Chapter 7

\title{
Brain Functional Networks in the Developing Brain Using Resting BOLD
}

\author{
Wei Gao, Hongtu Zhu, Kelly S. Giovanello, \\ J. Keith Smith, Dinggang Shen, John H. Gilmore and \\ Weili Lin
}

Additional information is available at the end of the chapter

http://dx.doi.org/10.5772/30640

\section{Introduction}

One of the most astounding transformations that takes place in the living world is the transformation of human brain from an earthworm like neural tube in the embryo to a well organized system consisting of billions of neurons, trillions of synapses, as well as supporting cells/structures. Equally astounding is the emergence of various complex functions that accompany this remarkable structural transformation. In the long query to shed light on brain's functional development process, two questions - (1) how and when specific functional modules emerge; and (2) how different functional modules integrate/ coordinate to form an efficient brain system, lie in the core. Traditional behavioral studies (Amsterdam, 1972; Cepeda et al., 2001; Diesendruck et al., 2004) have provided valuable information about the developmental process, but such studies do not offer insights into the anatomical and functional development of the brain in relation to the observed behavioral maturation. Neuroimaging studies, in contrast, (including positron emission tomography (PET), magnetic resonance imaging (MRI), etc) enable either direct or indirect elucidation of the underlying neuronal activity, potentially, unveiling the neurophysiological mechanisms guiding the developmental process. Insights from such studies are also critical for better understanding of the neuropathology underlying various developmental disorders, whose early diagnosis/intervention/treatment may benefit greatly from the quantitative and objective nature of these investigations.

Traditional task-based functional magnetic resonance imaging (fMRI) techniques have been a powerful and non-invasive tool for adult brain imaging for decades (Bandettini et al., 1992; 
Belliveau et al., 1991; Ogawa et al., 1990) and its application in developmental investigation has also been long practiced. The feasibility of fMRI in newborns was first demonstrated by Born et al in 1996 (Born et al., 1996), who reported a differential visual activation pattern between infants and older children. Next, Hykin et al (Hykin et al., 1999) successfully conducted fMRI experiments in fetuses in 1999. Other groups have subsequently reported different functional activation patterns during both pre-and postnatal periods (Anderson et al., 2001; Born et al., 1998; Dehaene-Lambertz et al., 2002; Fulford et al., 2003). However, constrained by the inability to "perform tasks," only visual, auditory or tactile stimuli could be applied in such investigations, greatly limiting the scope of brain functions that were explored using this technique.

The recent advance of resting state functional connectivity MRI (fcMRI) technique (Biswal et al., 1995; Lowe et al., 1998), however, has greatly expanded the scope of fMRI beyond taskbased approaches and provided a unique opportunity to answer fundamental questions about the brain's functional network organization. Initially described in Biswal et al's seminal paper (Biswal et al., 1995), fcMRI measures temporal synchronization of spontaneous blood oxygen level dependent (BOLD) fluctuations at rest, eliminating the need for external stimuli/tasks. Thus, fcMRI greatly simplifies the experimental design and provides unique information regarding the brain's functional organization that is not available through traditional taskbased fMRI analysis. In adult subjects, various resting state functional networks (RSNs) have been consistently reported including a motor-sensory network (Biswal et al., 1995), a visual network (Lowe et al., 1998), attention networks (Fox et al., 2006), a memory network (Vincent et al., 2006), and a network mediating self-related thinking (i.e., the brain's default-mode network) (Greicius et al., 2003; Raichle et al., 2001; Shulman GL, 1997). Results from these RSNs are highly consistent with the known anatomical connection and/or functional coactivation patterns (Biswal et al., 1995). More importantly, fcMRI is particularly suited for studies where the target population is not able to perform specific cognitive tasks as required by traditional fMRI, such as studies of early brain development and neurological diseases (Greicius et al., 2004; Liu et al., 2008; Seeley et al., 2009).

Although fcMRI has been successfully applied in numerous studies aimed at unveiling the brain's functional organization (e.g., development, aging, disease), several issues could affect the robustness/interpretation of the obtained results. Among them, the physiological underpinnings of fcMRI signal remain elusive. Since the BOLD signal provides only an indirect measure of the underlying neuronal activity, a pressing need for understanding of the neuronal correlates of the fMRI signal has been widely recognized. The consensus is that task-driven BOLD signal is highly correlated with local field potential (LFP), which is a complex signal arising from integrated electrical activity in the pre-and postsynaptic terminals recorded in the vicinity of embedded microelectrodes in the cortex (Logothetis, 2003; Logothetis et al., 2001; Raichle and Mintun, 2006). However, neuronal dynamics resulting from external tasks are likely fundamentally different from that observed in fcMRI signal. As a result, a direct translation from the previous findings of task-driven fMRI to fcMRI interpretation may be untenable. To directly delineate the underlying neural correlates of spontaneous BOLD signal fluctuations, several groups have made important discoveries. First, using an anesthetized 
monkey model, Shmuel and Leopold (Shmuel and Leopold, 2008) demonstrated a significant, time-lagged correlation between spontaneous BOLD fluctuations and the power of neuronal activity in the local filed potential gamma band, multi-unit activity, and spiking activity, directly supporting the notion that fcMRI correlation reflects the underlying neuronal interplay between remote cortical regions. Second, by extracting signal from the depth electrodes placed in the auditory cortex of epilepsy patients, Nir et al (Nir et al., 2008) found significant inter-hemispheric correlations in the slow modulations of neuronal firing rates and gamma (40 100Hz) LPF power with a clear spatial selectivity, further revealing the underlying neuronal correlates of fcMRI signal. Additionally, He et al (He et al., 2008) also examined the correlation structure of slow cortical potential (SCP) signal recorded from the implanted electrodes in a similar epilepsy patient study and showed a consistent correlation structure with that from fcMRI signals, providing evidence of the possible contribution of SCP in the observed fcMRI correlation structure. Overall, although some discrepancies exist among the studies examining the neuronal correlates of the observed fcMRI signal, the common finding of significant neuronal activity correlation strongly supports the notion that functional organization structures observed in fcMRI experiments reflect underlying neuronal interplay. Future studies are still needed to further discern the physiological origins of fcMRI signal.

While fcMRI has been utilized to examine a wide variety of neurological diseases (Fair et al., 2010; Greicius et al., 2004), aging (Achard and Bullmore, 2007), as well as brain development (Fair et al., 2008; Fransson et al., 2007; Gao et al., 2009), emphasis in this chapter is given to how fcMRI can be used to explore temporal and spatial evolution of early brain functional development. In particular, we will focus on the first two years of life - the most critical time period for postnatal brain development (Tau and Peterson, 2010). As early as the fifth week after conception, the first synapses begin forming in the fetus's spinal cord which permits the first fetal movements such as spontaneous arches and curls of the whole body (Joseph, 1999). More complicated and coordinated movements such as stretching, yawning, sucking, swallowing, and grasping soon follow in the first and second trimesters. Toward the end of the prenatal period, fetuses begin to respond to familiar odors and sounds (Joseph, 1999). It is clear that nature ensures such vital and basic bodily functions are in place by the time a baby emerges from the supporting womb.

After prenatal development, the first two years of life are commonly viewed as the most critical time period for postnatal brain development (Tau and Peterson, 2010). At birth, the brain is approximately one-quarter of its adult size and quickly grows to an adult-like volume (around $80 \%$ ) by the end of the second year. The dramatic increase in the number of dendrites and synapses is partly responsible for such a fast volume increase. In fact, the first two years of life are an exuberant period for synapse growth and cerebral cortex produces most of its synaptic connections. By the end of the second year, a toddler's cerebral cortex contains more than one thousand trillions of synapses, which are far more than that in an adult's brain (around 50 percent more) and will experience a prolonged, experiencedependent pruning process to achieve its matured configuration (Johnson, 2000, 2001; Tau and Peterson, 2010). Axonal elongation, thickening, and myelination processes are also important growth factors during this period (Gao et al., 2008; Haynes et al., 2005). It has been documented that long axonal 
connections are nearly complete around 9 months of age (Conel, 1939-1963; Tau and Peterson, 2010). Moreover, continuous myelination of white matter during the first two years of life greatly improves the brain's electrical signal transfer efficiency (Haynes et al., 2005). Concurrent with the above mentioned dramatic structural development is the parallel improvement in brain function. Decasper and Fifer (DeCasper and Fifer, 1980) demonstrated that the sensorimotor experiences of fetus and voice of mother (Kolata, 1984) can be recognized. Additionally, Fivush and Hamond (Fivush, 1990) showed that 2yr olds can already retrieve much detail about a trip to the zoo. Studies on toddlers also revealed that 18-to 24-month-olds are able to use speaker's gaze direction (Baldwin, 1993) and affective expression (Tomasello et al., 1996) as cues leading to speaker's communicative purposes. Akhtar and Tomasello (Akhtar, 2000) further proposed that children are able to infer the meaning of words through an understanding of people's minds (Diesendruck et al., 2004). More importantly, one of the unique properties of human self-awareness has also been documented to undergo major development during the first two years of life (Amsterdam, 1972). The gradual emergence of such higher order cognitive functions leads to the hypothesis of parallel establishment of corresponding functional networks during this critical period. Moreover, as more complex functions emerge, the brain's information transfer efficiency is also expected to improve. Leveraging on graph theory analysis (Bullmore and Sporns, 2009), brain's information transfer efficiency can be effectively measured based on fcMRI time series data.

Although this chapter primarily focuses on the applications of fcMRI in normal brain development, fcMRI studies in developmental disorders are also briefly reviewed. This chapter is organized as follows: Section 2 provides a review of data acquisition, preprocessing and various analysis approaches for fcMRI study; Section 3 illustrates recent findings on the emergence of various functional networks; Section 4 shifts attention from individual networks to the whole brain network; Section 5 reviews recent applications of fcMRI on various developmental disorders; Section 6 provides a discussion of the limitations and future directions of the use of fcMRI in developmental studies; and finally, Section 7 provides a conclusion.

\section{Data acquisition and analysis}

The acquisition of resting state fcMRI data is identical to that of traditional task-based fMRI (except no task/stimuli are required during the acquisition) for which echo planer imaging (EPI) is the most frequently used imaging sequence due to its fast scan time. Using an EPI sequence, a 2D slice can be acquired in approximately 40 to $100 \mathrm{~ms}$ and 2 to 3 seconds for whole brain coverage, which alleviates motion-related artifacts during imaging. However, this sequence is prone to other artifacts related to either hardware (e.g., eddy current effect) or imaging sample (e.g., T2* related susceptibility effect). Moreover, although EPI is already a fast imaging technique, the time resolution ( 2 seconds) is still far from sufficient to characterize the dynamic of underlying neuronal activity and unable to faithfully sample several physiological factors such as respiratory/cardiac cycles resulting in potential noise contamination. Therefore, further improvement in imaging acquisition methods for faster scanning is 
greatly desired (Feinberg et al., 2010). Another important factor in fcMRI experiments is the required duration of acquisition. Van Dijk et al. (Van Dijk et al., 2010) compared the correlation strengths resulting from different lengths of data acquisitions (from $\sim 4 \mathrm{~min}$ to $\sim 12 \mathrm{~min}$ ) and reported that they stabilized at 5-6 min of data acquisition. More recently, Whitlow et al. (Whitlow et al., 2011) reported similar findings and further proposed that graph theory metrics of network connectivity might be accurately calculated from as little as 1.5 2 min of fcMRI data. Besides these technical considerations, fcMRI study requires that subjects are at a "resting" state. In adult studies, different ways of keeping subjects at resting are utilized such as fixation, eyes closed resting (ECR), and eyes open resting (EOR). Van Dijk et al. (Van Dijk et al., 2010) compared the functional connectivity strength within default and attention networks during these different resting states and reported significant differences between ECR and fixation, as well as between ECR and EOR, but not between EOR and fixation, indicating a significant effect of eyes opening versus eyes closing. However, similar comparisons on other functional networks are lacking and the conclusion might not be directly generalized. In developmental studies, keeping the subjects "resting" and still within the scanner at wake condition proves to be extremely challenging. Therefore, most developmental studies, especially those focusing on the first two years of life, scan their subjects during sleeping state (either natural sleeping or sedation-induced sleeping). For older children, as keeping them sleeping within the scanner becomes harder, watching movie is an alternative for keeping them still in the scanner, but the brain state difference between sleeping and movie watching should be considered (Gao and Lin, 2012).

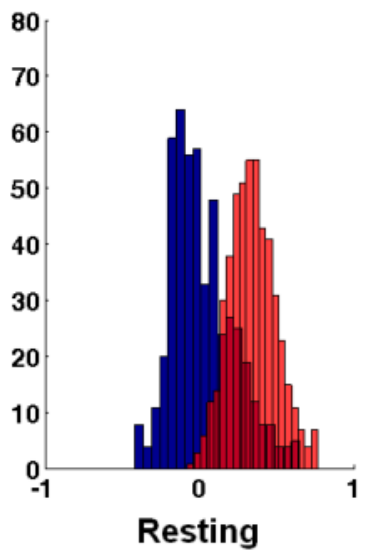

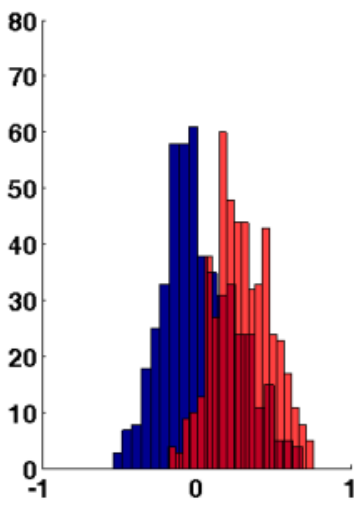

Finger Tapping

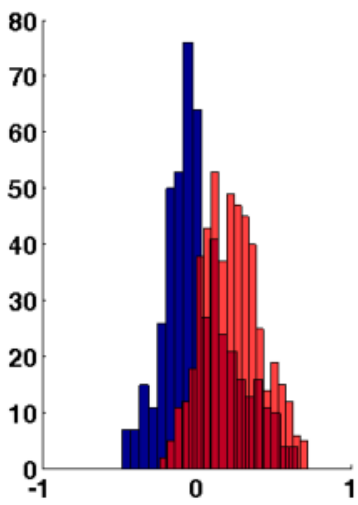

Movie Watching

\section{- With global signal regression Without global signal regression}

Figure 1. The histograms of the group mean correlation matrices (16 subjects, 32 ROIs covering five predefined functional networks, 496 correlations) with (blue) and without (red) global signal regression during three experimental conditions-resting, finger tapping, and movie watching. This figure was reproduced with the permission from Gao and Lin. (2012). 
While acquiring fcMRI images is rather straightforward and EPI is the most widely employed imaging sequence, the means through which fcMRI data is processed to discern brain functional connectivity is less consistent across different research labs. Typically, fcMRI is preprocessed, including time shifting, motion correction, spatial smoothing, linear trend removal, low pass filtering (typically $<0.1 \mathrm{~Hz}$ ) and nuisance signal regression. Although minor discrepancies exist regarding the pre-processing procedures among different researchers, most of the steps are fairly consistent and widely accepted with the exception of nuisance signal regression, especially global signal removal, which is designed to minimize the potential sources of physiological noise such as respiration and cardiac motion (Chang and Glover, 2010; Fox et al., 2009; Murphy et al., 2009). Such a regression forces the resulting correlation strength distribution (from a seed-based analysis) to be bellshaped centered at zero. Fig. 1 shows the distribution of correlation coefficients with and without global signal removal during three different experimental conditions-resting, finger tapping, and movie watching (Gao and Lin, 2012). It is immediately apparent that the removal of global signal leads to the presence of negative correlations. One immediate question is whether the observed negative correlation is "introduced" by global signal removal or is "brought" into light after removing the nuisance factors (global signal) obscuring the true relationships. Great controversy exists concerning the interpretation of negative correlations (Fox et al., 2009; Murphy et al., 2009). Fox et al (Fox et al., 2009) have shown that after removing the networks in question from the global signal mask (essentially eliminating the mathematical constraints on the two systems), certain anticorrelation can still be observed. Moreover, in an independent study, Chang and Glover (Chang and Glover, 2010) showed evidence of the presence of anti-correlation between the default network and the task-positive dorsal attention network, both with and without controlling for physiological noise variation induced by respiration and heart beating (although physiological noise correction did increase the spatial extent and magnitude of negative correlations). Such findings partially support the "authenticity" of the observed negative correlation, but future studies are needed to further delineate the nature of the global signal and the interpretation of the negative correlations after their removal.

After the pre-processing steps, three categories of methods generally exist for further processing fcMRI data to examine brain functional connectivity: (1) seed-based method; (2) independent component analysis based method; and (3) graph-theory analysis based method. The seed-based approach is the most widely adopted likely due to its simplicity in both implementation and interpretation. By designating a seed region for obtaining the reference signal, a voxel-wise whole brain correlation analysis can be conducted to acquire individualbased functional connectivity maps, which can then be pooled together to obtain a group map after statisitcal testing of each voxel's connectivity strength. Such a map represents the architecture of the functional network dictated by the seed region, enabling easy interpretation of the results. However, this approach could potentially be biased by the choice of the seed region and can only determine the brain functional connectivity specifically associated with the chosen seed. Most of the reported studies detemine the seed region either based on anatomical features or published coordinates of previous fMRI activation studies. Then a spherical ROI is defined centered at the seed with a certain radius, where both the location and size can vary from study to study. This variability poses a potential problem for direct 
comparisons between different studies. This problem is worsened in developmental studies since both the structural and functional brain organization might differ from those of adults, making the choice of seed regions based on the readily available adult literatures impractical. Mathematically, seed-based correlation analysis measures only the pair-wise correlation between a seed region and the voxel in question while ignoring the multivariate relationship among all brain voxels, which may be suboptimal especially when one intends to define the architectures of several networks simultaneously (Joel et al., 2011).

The independent component analysis (ICA) approach, on the other hand, largely alleviates the above mentioned problems. First, since it does not need pre-specified seed regions but extracts spatially/temporally independent components in a data-driven fashion, the biases associated with choosing seed regions are largely avoided. Second, depending on whether spatial or temporal ICA is applied, this approach maximizes the spatial/temporal independence among all identified components simultaneously by considering all voxel's time courses together, thus alleviating the "pair-wise" correlation problem embedded in the seed-based approach. Given these advantages, numerous studies (Damoiseaux et al., 2006; Fransson et al., 2010; Gao et al., 2009) have applied ICA and the results are fruitful. However, these advantages come at a cost. Since there is no inherent "ranking" available for ICA-extracted components, selecting components reflecting brain functions is a matter of subjectivity through either visual inspection or template matching. Although quantitative methods (De Martino et al., 2007; Perlbarg et al., 2007; Tohka et al., 2008) aiming to solve this issue are emerging, their effectiveness and robustness need to be validated by independent studies.

Another widely used strategy is to parcellate the entire brain into a set of ROIs and to perform graph-theory based analyses to delineate the whole brain's functional topology. More detailed reviews of graph theory based analysis can be found in Bullmore and Sporns (Bullmore and Sporns, 2009). The small-world related measures (Latora and Marchiori, 2001; Watts and Strogatz, 1998) are especially popular and have been used to quantify the brain's development (Supekar et al., 2009), aging (Achard and Bullmore, 2007), as well as various disease processes (Liu et al., 2008). However, similar to a seed-based approach, there is no standard approach as to how the whole brain should be parcellated. In practice, different anatomical feature based parcellation schemes including AAL (Tzourio-Mazoyer et al., 2002), ANIMAL (Collins et al., 1995) have been used. However, as described by Fornito et al (Fornito et al., 2010) and Wang et al. (Wang et al., 2009), different graph analytic measures may arise from different parcellation resolutions, making direct comparisons between studies untenable. Moreover, anatomical parcellation approaches do not explicitly consider functional similarity within the defined regions. As a result, spatial intra-region inhomogeneity of the BOLD signals may lead to spuriously averaged signals, and hence, inaccurate graph analytic measures. Data driven functional similarity based brain parcellation schemes are particularly desired in studies where the architecture of brain functional regions may deviate from that in normal adults. Such methods (Nelson et al., 2010; Neumann et al., 2006; Shen et al., 2010) directly utilize Pearson's correlation or canonical correlation between BOLD time series as similarity measure aiming at defining functionally sensible parcellations. As such strategies could effectively address the functional inhomogeneity concern inherent in anatomical-defined parcellation schemes, 
potential issues also exist. Among them, the most challenging one might be the number of regions to define. As most current methods relying on predefining a fixed number based on prior information, more data-driven approaches are highly desired and deserve further effort.

In addition, another limit for graph-theory based methods is that there is currently no optimal way to compare networks with different number of nodes and/or edges (van Dijk et al., 2010). A summary of different fcMRI analysis methods can be found in Table. 1.

\begin{tabular}{|c|c|c|c|}
\hline Methods & Brief Description & Advantage & Disadvantage \\
\hline Seed-based method & $\begin{array}{l}\text { By designating a seed region } \\
\text { for obtaining the reference } \\
\text { signal, a voxel-wise whole } \\
\text { brain correlation analysis is } \\
\text { conducted to define a } \\
\text { particular functional } \\
\text { network. }\end{array}$ & $\begin{array}{l}\text { Easy implementation ; } \\
\text { Straightforward } \\
\text { Interpretation. }\end{array}$ & $\begin{array}{l}\text { Seed choice bias; Focusing } \\
\text { on pair-wise correlation } \\
\text { while ignoring multivariate } \\
\text { relationship among multiple } \\
\text { networks. }\end{array}$ \\
\hline $\begin{array}{l}\text { Independent component } \\
\text { analysis (ICA) }\end{array}$ & $\begin{array}{l}\text { Functional networks are } \\
\text { extracted through blind } \\
\text { source separation technique- } \\
\text { ICA. }\end{array}$ & $\begin{array}{l}\text { Data-driven way to define } \\
\text { functional networks; } \\
\text { Simultaneous extraction of } \\
\text { multiple functional networks } \\
\text { based on their multivariate } \\
\text { relation. }\end{array}$ & $\begin{array}{c}\text { Subjective selection of } \\
\text { independent components for } \\
\text { subsequent analysis. }\end{array}$ \\
\hline Graphtheory based analysis & $\begin{array}{l}\text { The brain is first pacellated } \\
\text { into a set of ROls after which } \\
\text { graphtheory based analysis is } \\
\text { performed to evaluate } \\
\text { brain's functional topology } \\
\text { using various network } \\
\text { measures. }\end{array}$ & $\begin{array}{l}\text { Whole brain analysis } \\
\text { enabling assessment of } \\
\text { brain's overall information } \\
\text { transferring property } \\
\text { through various graph- } \\
\text { theory based measures. }\end{array}$ & $\begin{array}{l}\text { Anatomically defined } \\
\text { parcellation; Currently no } \\
\text { standard ways to compare } \\
\text { networks with different } \\
\text { numbers of nodes and/or } \\
\text { edges. }\end{array}$ \\
\hline
\end{tabular}

Table 1. Typical methods applied in fcMRI study.

\section{The emergence of individual functional networks during the first two years of life}

A mature brain works as a unified system involving various functionally specialized networks each dealing with an array of specialized functions. Generally, depending on the nature of the functions, brain networks are classified into primary functional networks such as sensory, motor, and visual networks or higher order cognitive networks such as attention, memory, executive control, emotion, etc. The developmental trajectory of each individual functional network is of great interest. Successful interaction with the outside world rests on the proper 
functioning of the primary networks and it has been suggested that they are essential for the tuning and establishment of the higher order cognitive networks (Johnson, 2000, 2001). Therefore, it is anticipated that primary functional networks will develop faster and earlier than those higher order cognitive functional networks.

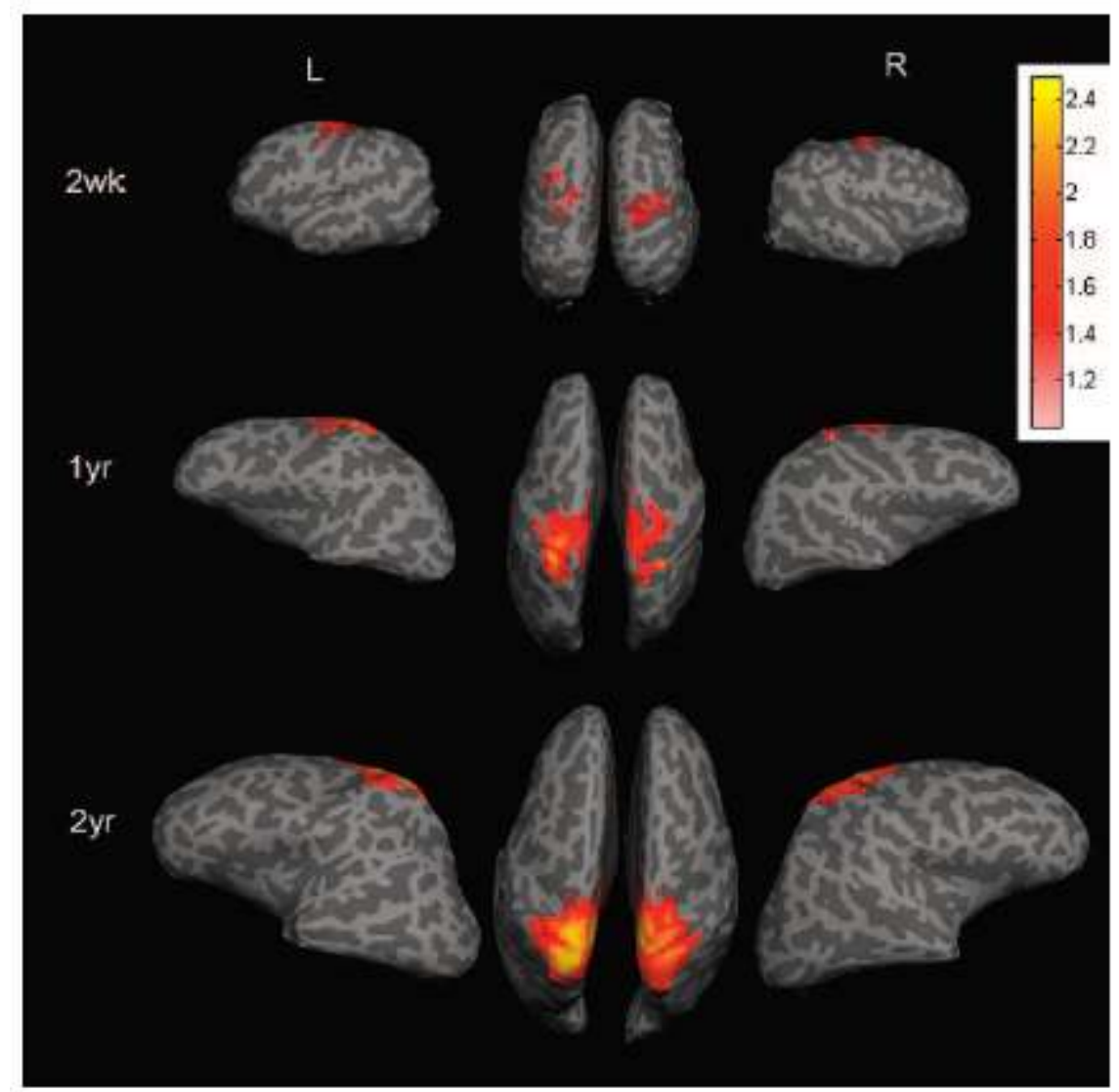

Figure 2. The functional connectivity maps of motor-sensory network at $2 \mathrm{wks}, 1 \mathrm{yr}$ and $2 \mathrm{yrs}$ of age, respectively. The color bar represents z-score values. This figure was reproduced with the permission from Lin et al. (2008).

Using a seed-based approach, we (Lin et al., 2008) have reported the developmental patterns of both the motor-sensory and visual networks during the first two years of life (Fig. 2 and Fig. 3). The seeds were manually drawn around bilateral motor-sensory cortex and visual cortex for the two networks, respectively. The most noticeable feature is that starting at neonates, the two networks already demonstrate a bilateral-symmetric functional connectivity pattern similar to that of $2 \mathrm{yr}$ olds, which is also reasonably consistent with their adult configuration (Biswal et al., 1995). Consistent with this finding, Fransson et al (Fransson et al., 2007) 
also reported the existence of adult-like motor-sensory, visual, as well as auditory networks in preterm infants scanned at term equivalent age. A longitudinal study by Smyser et al (Smyser et al., 2010) further delineated the dramatic development of such primary sensory networks during the prenatal period from 26 weeks postmenstrual age to the term equivalent age supporting the network structures observed in neonatal subjects. The early synchronization of these primary sensory functional networks most likely indicates an evolutional optimization to ensure early survival as well as effective interaction with the outside world for proper experience-based tuning of other higher order networks. However, despite of the common feature of early synchronization, Fig. 2 and 3 show that the motor-sensory network demonstrates more development during the first year of life, while visual network shows more increase during the second year of in terms of normalized volume of cortical connectivity, which indicates certain level of differential growth trajectories for different primary networks.

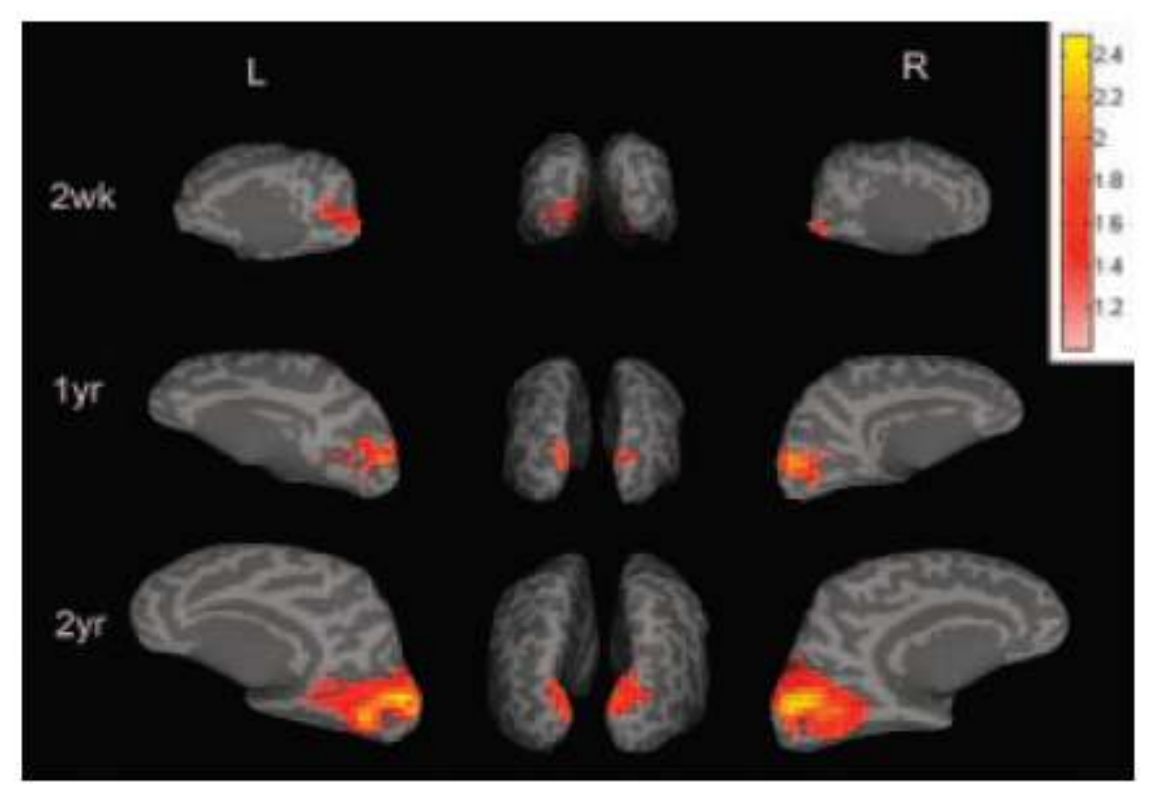

Figure 3. The functional connectivity maps of visual network at $2 \mathrm{wks}, 1 \mathrm{yr}$ and $2 \mathrm{yrs}$ of age, respectively. The color bar represents z-score values. This figure was reproduced with the permission from Lin et al. (2008).

While the primary brain functional networks appear to be reasonably developed immediately after birth, the growth trajectories of other higher order cognitive functional networks likely differ. Recently, a novel brain functional network was proposed by Raichle et al (Raichle et al., 2001) where the involved brain regions exhibit down-regulated activity during various goaldirected external tasks but up-regulated activity during uncontrolled resting state (Shulman GL, 1997). Various studies have been conducted in an attempt to probe the exact function of this network (Buckner et al., 2008; Buckner and Carroll, 2007; Greicius and Menon, 2004; Gusnard et al., 2001; Gusnard and Raichle, 2001). Among them, the consensus is that this 
network mainly supports self-related internal cognition (Andrews-Hanna et al., 2010; Buckner et al., 2008; Buckner and Carroll, 2007; Gusnard et al., 2001). From a developmental perspective, the establishment of self-consciousness has to be the preface of such self-referential thinking process and hence is likely related to the integrity of this network. Interestingly, Amsterdam (Amsterdam, 1972) vividly documented the emerging process of self-consciousness during the first two years of life. He showed that neonates could not recognize themselves from their mirror images but infants from 6 through 12 months of age demonstrate prolonged and repeated reaction to their mirror images as a sociable playmate. Wariness, withdrawal, selfadmiring, and embarrassed behaviors start at 14 months and have been observed in $75 \%$ of the children after 20 months of age. From 20 to 24 months of age, the majority of subjects demonstrate recognition of their mirror images. If the default network is indeed responsible for the self-consciousness based internal thinking process, we would expect a gradual orchestration of this network during the first two years of life. Fig. 4 shows the default network of $3 \mathrm{wks}, 1 \mathrm{yr}$ and $2 \mathrm{yr}$ olds, respectively. As shown, a rather primitive/incomplete default network consisting of 6 brain regions is observed in neonates, among which only medial prefrontal cortex (MPFC) and posterior cingulate cortex (PCC) regions are typically observed in its adult configuration. The finding of an "incomplete" default network is consistent with results from several other studies (Fransson et al., 2007; Smyser et al., 2010). However, at 1yr old, a total of 13 regions are observed and 10 of them are consistent with that observed in adults, including ventral/dorsal MPFC, PCC/retrosplenial (Rsp), bilateral lateral temporal cortex (LTC), bilateral inferior parietal lobule (IPL), and the hippocampal formation (HF) (Buckner et al., 2008). Such dramatic synchronization reflects fast orchestration of this network during the first year of life. In $2 \mathrm{yr}$ olds, the default network continues to grow by covering more regions (13 regions) consistent with adult's configuration. However, 6 non-typical regions including the orbital frontal, anterior cingulate cortex (ACC), right and medial parietal, and bilateral superior temporal regions are also observed.

The spring embedding plots (Ebbels et al., 2006) further demonstrate the topology of the default network throughout the first two years of life (Fig. 5). These graphs are arranged such that the most strongly connected regions were drawn close to each other and placed in the center of the graph and vice versa. One notable feature is that both PCC/Rsp and MPFC are consistently located in the center of each graph with the exception of the neonate group (only PCC/Rsp), implying that these two regions are most strongly connected with other regions. Moreover, the PCC/Rsp and MPFC reliably exhibit the highest mean connection strengths across all ages (Fig. 6). Such finding is consistent with adult studies (Buckner et al., 2008) reporting the "hub" role of these two regions in this distributed network. The betweenness centrality (BC) (Brandes, 2001) - a measure of node importance in graph theory further confirm that PCC/RSp and MPFC indeed serve as the hubs in the pediatric default network (Fig. 7). These results suggest that the PCC/Rsp may be the major hub of the default network whereas the MPFC subsequently emerges, potentially, as the secondary hub starting at 1yr of age.

Overall, a gradual emergence of the default network is observed during the first two years of life and the PCC/Rsp and MPFC emerge as two potential hubs. This finding is consistent with previous reports of the emergence of self-consciousness (Amsterdam, 1972), as well as other 


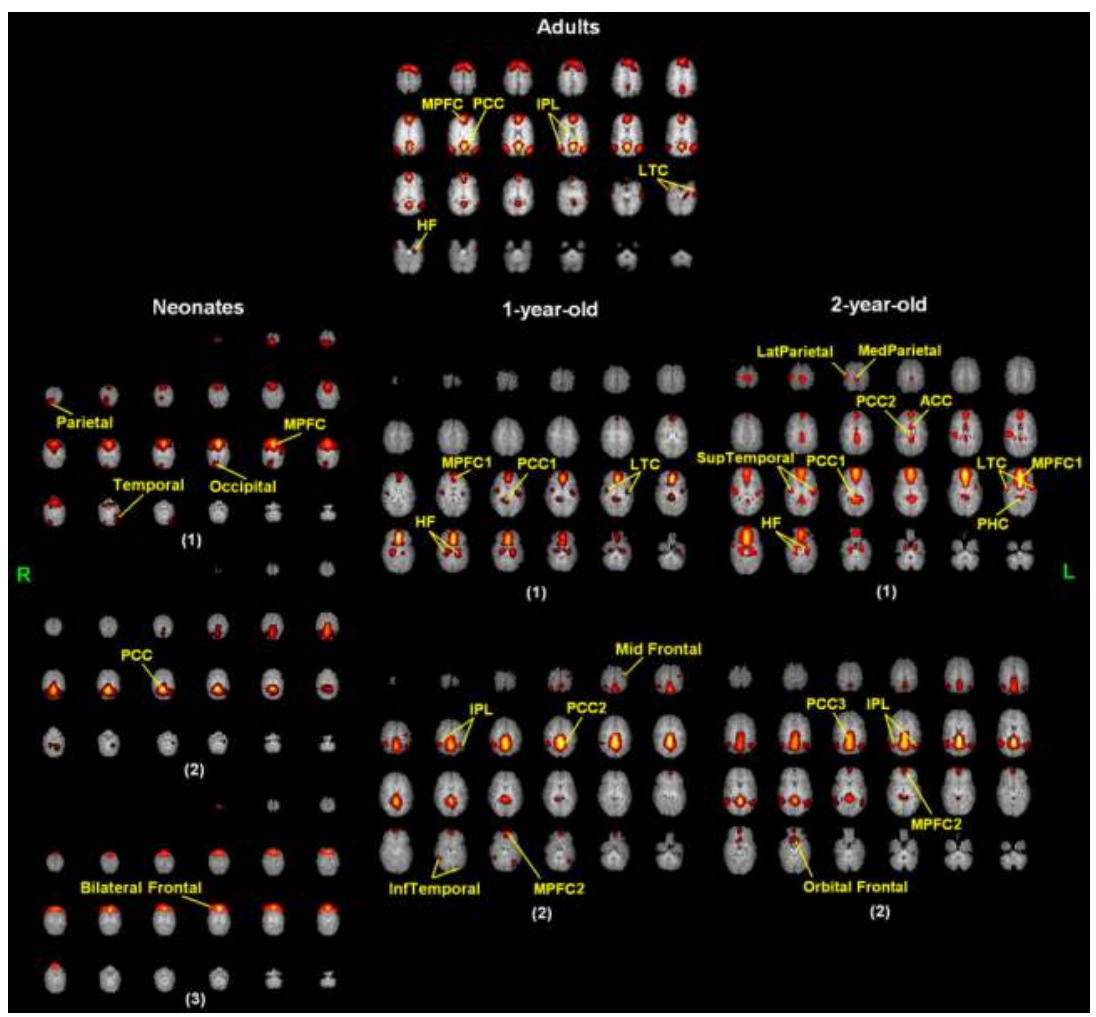

Figure 4. The independent components associated with brain's default network for neonates, $1 \mathrm{yr}$ olds, 2yr olds and adults. Individual regions are labeled for each map. ACC: anterior cingulate cortex; HF: hippocampal formation; InfTemporal: inferior temporal cortex; IPL: inferior parietal lobule; LTC: lateral temporal cortex; LatParietal: lateral parietal cortex; MedParietal: medial parietal cortex; MidFrontal: middle frontal cortex; MPFC: medial prefrontal cortex; PCC: posterior cingulate cortex; PHC: para-hippocampal cortex; SupTemporal: superior temporal cortex. This figure was reproduced with the permission from Gao et al. (2009).

default network related functions such as episodic memory (Fivush, 1990; Kolata, 1984). Moreover, the appearance of non-typical regions in all three pediatric groups' default network indicates further pruning/specialization process needed for final maturation. Therefore, in contrast to the primary networks (e.g., motor-sensory, visual) for which the major development is done prenatally, the establishment of the higher order default network takes prolonged postnatal development to achieve its functioning configuration.

Up to now, the discussions are largely focused on individual network level. As our brain is an integrated system consisting of many functionally specialized networks, it is of critical importance to further understand the interaction among different networks. Five networks including the previously described motor-sensory, visual, default network and two other higher order networks-dorsal attention and fronto-parietal control network (Vincent et al., 2008) were simultaneously studied based on an extended dataset including 51 neonates (27 

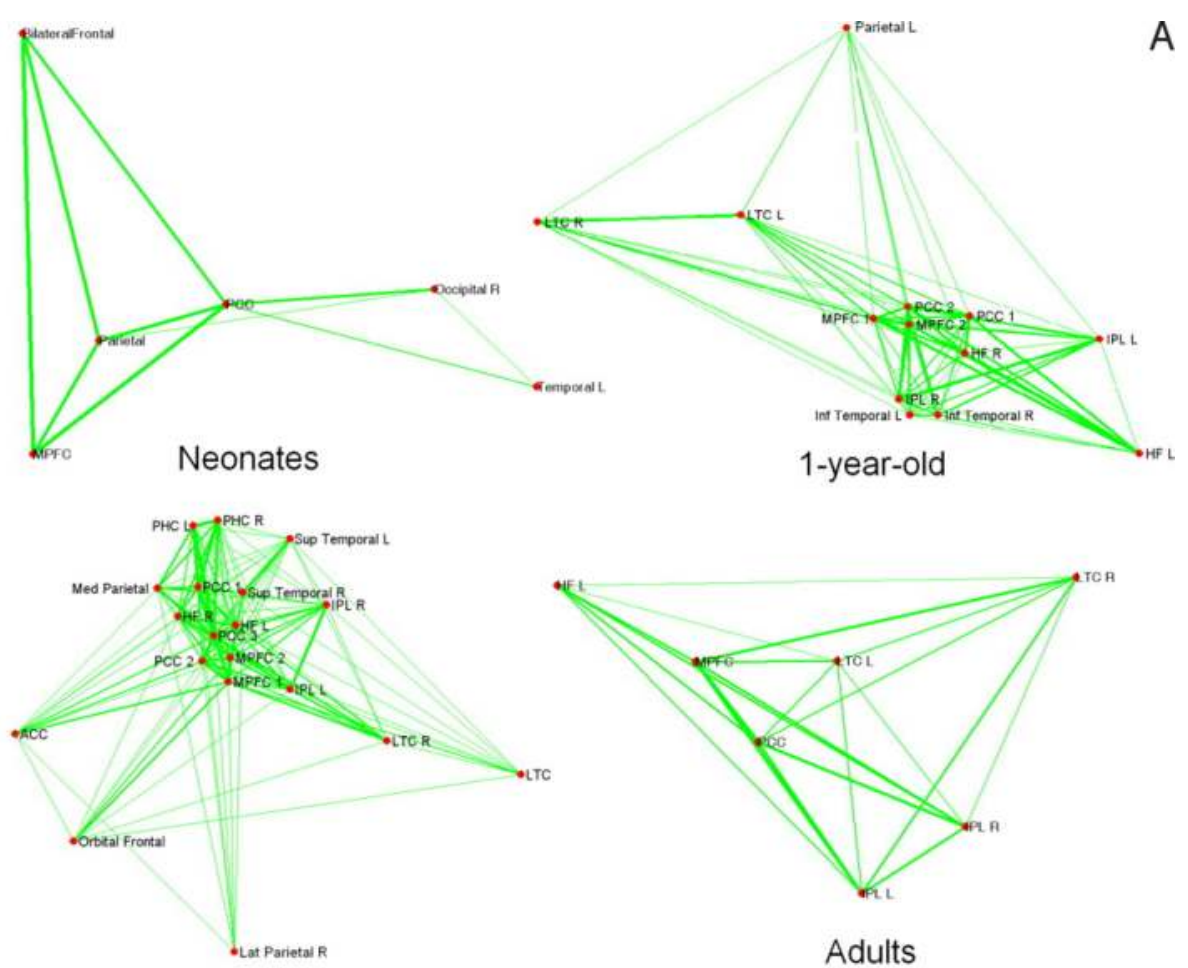

Adults

2-year-old

\section{Functional connectivity graph}

Figure 5. The spring embedding plots of the group-mean functional correlation patterns within the default network for neonates, 1yr olds, 2yr olds and adults. This figure was reproduced with the permission from Gao et al. (2009).

male, $23 \pm 12$ days (SD)), $501 \mathrm{yr}$ olds (27 male, $13 \pm 1$ months) and 46 2yr olds (28 male, $24 \pm 1$ months). Specifically, 5, 6, 6, 6, and 9 ROIs (8mm-radius sphere, altogether 32 ROIs) were defined based on the MNI coordinates published in adult studies (Vincent et al., 2008) to construct the five networks, respectively, from which the group mean correlation matrices were constructed $(32 \times 32)$ and visualized using spring-embedding plot (Fig. 8). In this figure, the segregation/synchronization of the five networks across the first two years of life can be simultaneously characterized. In neonates, except for the motor-sensory network (red) and the visual network (blue), which are already well organized into cohesive clusters, the remaining three higher-order cognitive networks are rather scattered (i.e., three separated clusters for default network (yellow); two for the dorsal attention network (magenta) and two for frontoparietal control network (brown).

This finding is highly consistent with results shown in Fig. 2-4, which again indicates early maturation of primary functional networks. In fact, the motor-sensory and visual networks 


\section{Neonates}

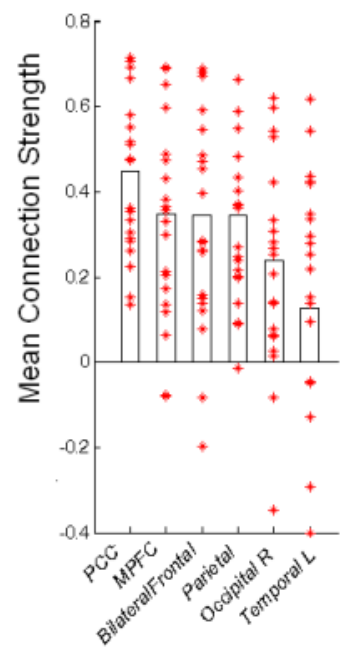

1-year-old

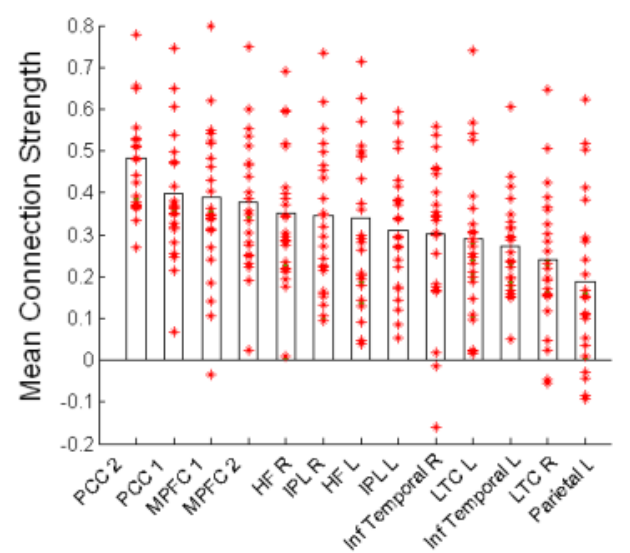

2-year-old

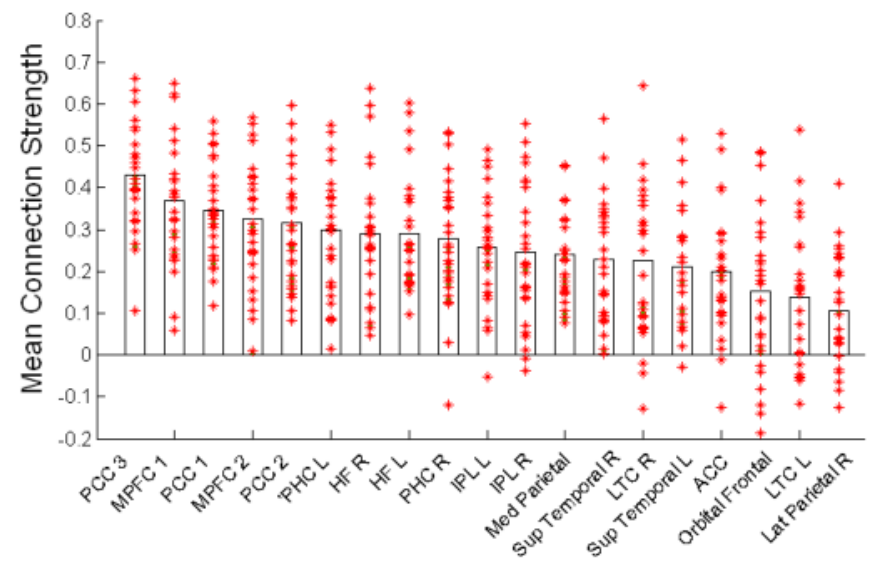

Adults

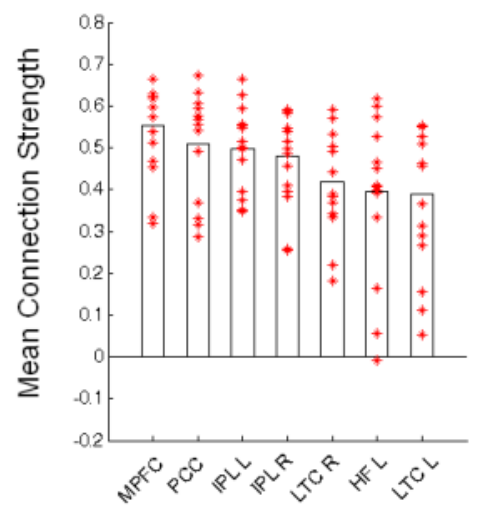

Figure 6. The mean connectivity strength of each node within the default mode network for neonates, $1 \mathrm{yr}$ olds, $2 \mathrm{yr}$ olds and adults. Each bar represents the group mean of each node's mean connectivity strength within the default network. Each asterisk represents an individual subjects' value. This figure was reproduced with the permission from Gao et al. (2009).

remain as cohesive clusters throughout the different age groups examined (i.e., neonates, $1 \mathrm{yr}$ olds, 2yr olds, and adults, Fig. 8). In great contrast, the synchronization of higher-order cognitive networks takes a more prolonged time. Specifically, the default network starts to be synchronized into one cluster in 1yr olds and remains so thereafter. However, although the other two networks (i.e., the dorsal attention network and the fronto-parietal control network) demonstrate certain long-distance synchronization during the first two years of life (Fig. 8), it 


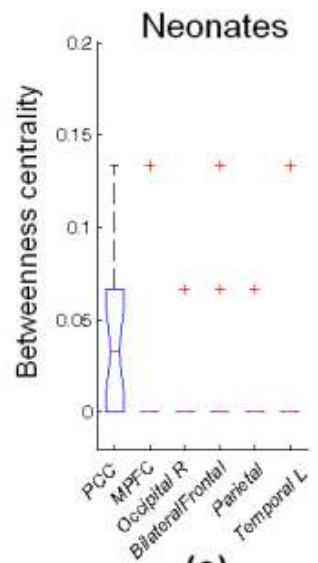

(a)

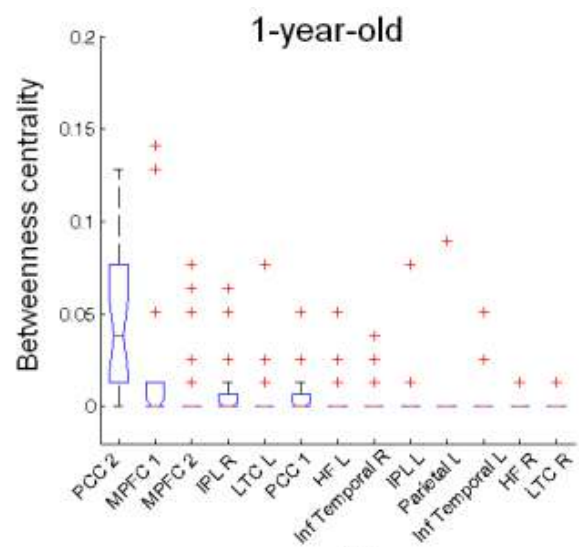

(b)

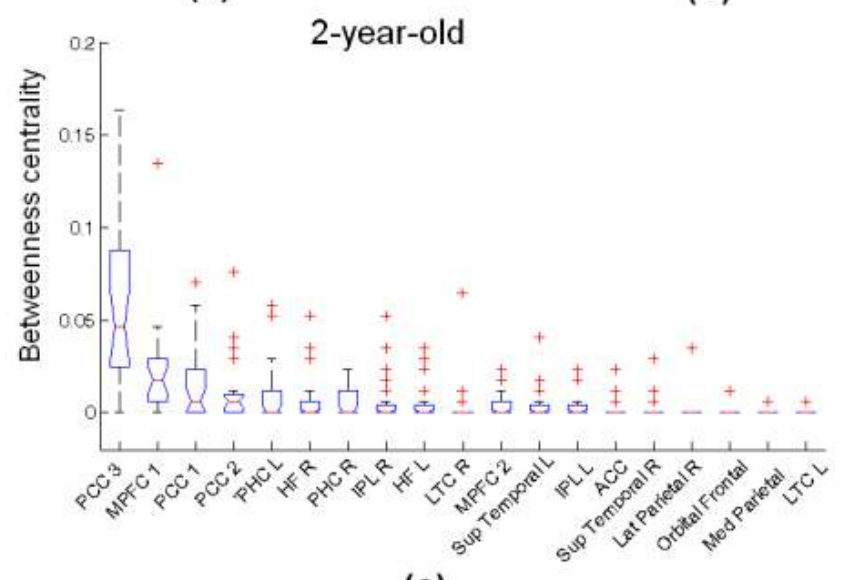

(c)

Figure 7. The betweenness centrality value for each node in the default network for neonates, $1 \mathrm{yr}$ olds, and $2 \mathrm{yr}$ olds. This figure was reproduced with the permission from Gao et al. (2009).

is not until adults are they organized into selfcontained clusters. Clearly, more quantitative characterization, as well as additional sampling between $2 \mathrm{yr}$ olds and adults, is highly desirable for more specific delineation of the complete growth trajectories of these five networks. Nevertheless, one trend is again clear in Fig. 8; primary networks such as motorsensory and visual are wired prenatally while the synchronization of higher-order cognitive networks is achieved during postnatal life (Fransson et al., 2007; Lin et al., 2008; Smyser et al., 2010). Interestingly, from a spatial perspective, higher order cognitive networks are usually spatially distributed but primary sensory networks are mostly locally wired. This local organization might partially contribute to their early synchronization. Specifically, it is likely that long distance connections are not established immediately after birth (discussed below) and that the anatomical proximity facilitates formation of short-distance connections so close 


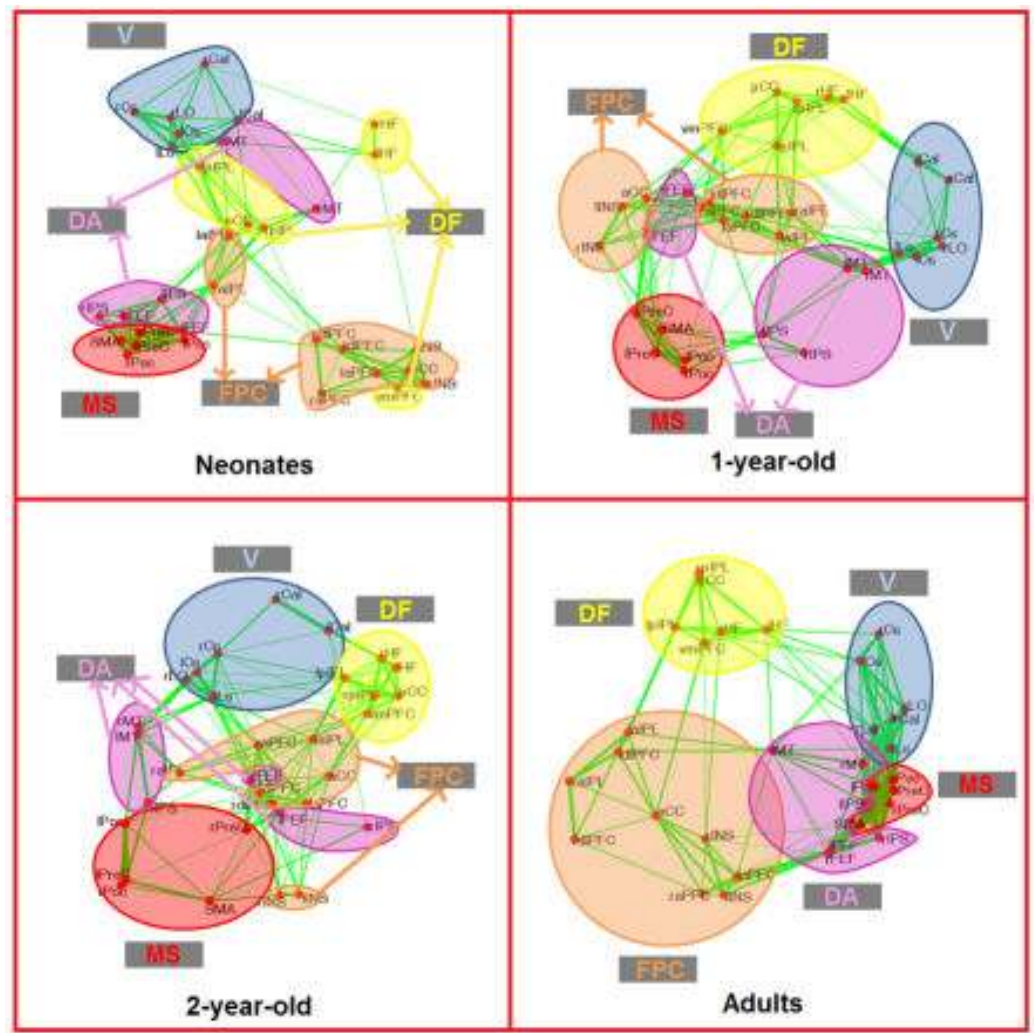

Figure 8. Spring embedding plots of the group-mean correlation pattern among five predefined functional networks: motor-sensory, visual, default, dorsal attention, and fronto-parietal control network. The spatial evolution of each network is highlighted by different colors. DA: dorsal attention network; DF: default network; FPC: fronto-parietal control network; MS: motor-sensory network; V: visual network.

interaction immediately after birth can be achieved. This property is likely attributed by evolutional optimization so that early survival can be ensured. Moreover, this evolution process promotes the development of experience based fine tuning and maturation of higher order networks (Johnson, 2000, 2001). Overall, existing results support the notion that brain's matured organization is optimized according to the developmental process which not only ensures early functioning of primary functions but also allows sufficient environmental tuning of higher order functions for better adaptation.

\section{The evolution of whole brain's efficient topology}

The examination of individual functional networks provides vital information about the early development of brain's sub-systems. Yet, how the whole brain topology evolves during this 
critical time remains elusive. By designating different brain regions as nodes and connections between them as edges, recent graph theory advancement, especially the smallworld topology (Watts and Strogatz, 1998) appears well suited to quantitatively characterize the complex network structure of the human brain. Small world topology is characterized by a high level of local cliquishness as well as sufficient long-range direct connections bridging the local clusters, leading to excellent local and global wiring efficiency for information transfer. The regions that bridge disparate and local clusters are important information transfer portals, usually termed network hubs (Barabasi and Albert, 1999). While many studies have confirmed the existence of small world characters in matured brain networks (Achard et al., 2006; Hilgetag et al., 2000; Salvador and E., 2005; Watts and Strogatz, 1998), Fransson et al (Fransson et al., 2010) showed that the infant (1 week old) brain also exhibits a small world topology. However, how the whole brain network topology continues to evolve during the first years of life remains elusive. In particular, given the dramatic axonal elongation/thickening and myelination during this period (Conel, 1939-1963; Fair et al., 2008; Tau and Peterson, 2010), the resulting enhancement in long-distance connections must have profound implications in achieving a highly efficient brain network. To test these hypotheses, 51 neonates ( 27 male, $23 \pm 12$ days (SD)), 50 1 yr olds ( 27 male, $13 \pm 1$ months) and 462 yr olds ( 28 male, $24 \pm 1$ months) with successful fcMRI scan were retrospectively identified and graph theoretical analyses were conducted (same as the data used in Fig. 7) (Gao et al., 2011).

A whole brain parcellation approach (as described above) was employed by parcellating the brain into 90 ROIs and correlation matrices from individual subjects were constructed after standard preprocessing (Section 2). Local efficiency (LE), global efficiency (GE) (Latora and Marchiori, 2001), and betweenness centrality measures (Brandes, 2001) were calculated. The significant correlation matrices ( $\mathrm{p}<0.05$, FDR corrected) are presented in Fig. 9 for neonates, $1 \mathrm{yr}$ and 2yr olds. Dramatically increased connection density (Fig. 9b) can be observed from neonates to 1yr olds. Moreover, the anatomical distance histogram plot (Fig. 9c) of these significant connections clearly demonstrates a rightward shift, suggesting increased longrange connections. More importantly, those connections exhibiting increased strength with age are always associated with significantly longer connection distances than those decreasing strength with age (Fig. 9d).

To further examine the topological changes as resulting from the emerging long-distance connections, the top $15 \%$ of most significant connections from the group mean correlation matrices were visualized on a brain surface in Fig. 10. Imposing a fixed cost ensures that the topological changes arise not from different number of connections but instead from the rearrangement of connections. Different nodes are classified into different functional modules (represented by different colors) based on modularity analysis of their functional relationship (Newman, 2006). Interestingly, the neonates group's modules are largely local and agree well with the boundaries of different lobes. However, 1yr and 2yr olds' modular structures become dramatically more distributed. For example, in 1yr olds, the cyan-colored module spans across temporal and parietal lobes; the red-colored module covers both the parietal and subcortical regions; while the magenta-colored module includes regions from the parietal, frontal, and temporal lobes. Similarly, the red-colored module covers parietal and subcortical regions; the 
(a)
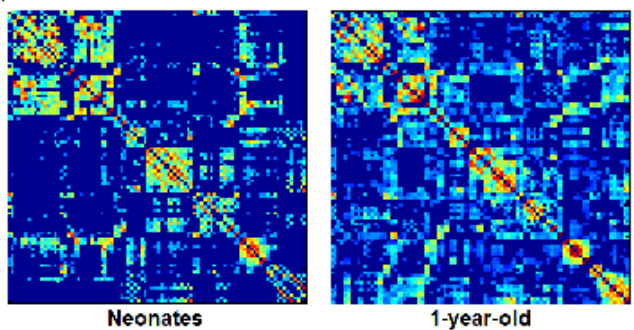

1-year-old
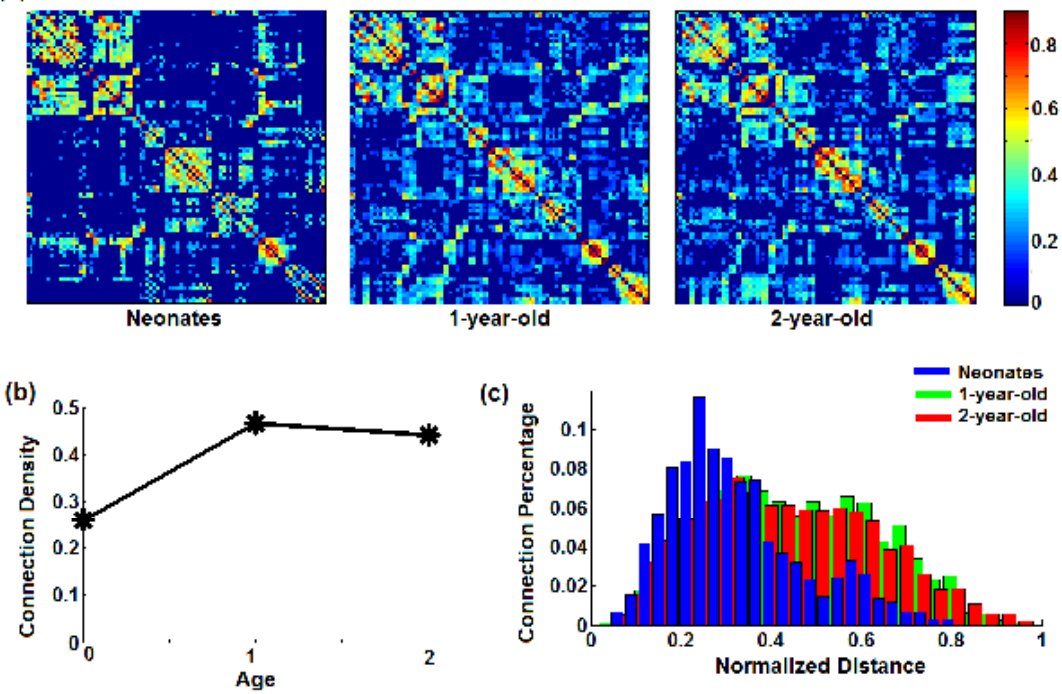

(c)

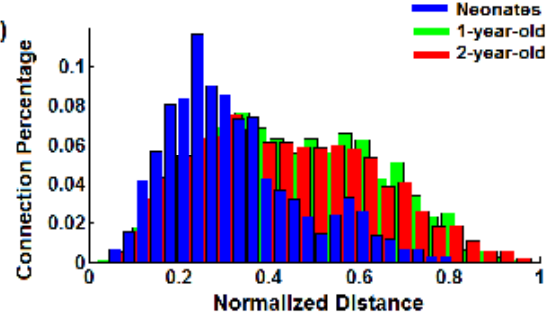

(d)
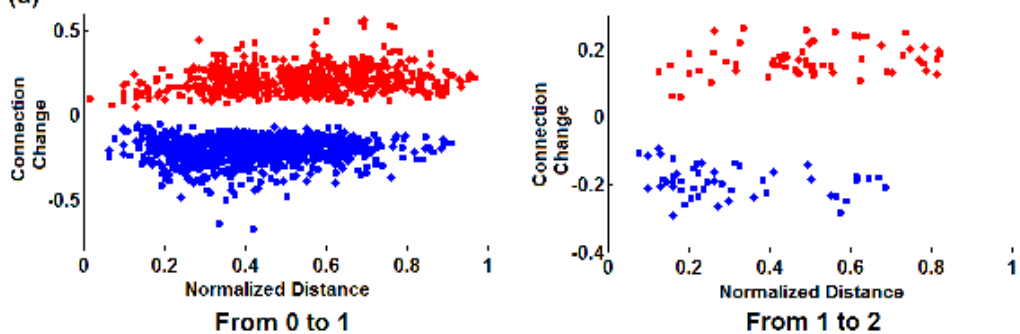

Figure 9. Assessment of age-dependent change of brain network topology. (a) significant connectivity matrices $(p<0.05$, FDR corrected) for neonates, $1 \mathrm{yr}$ and 2yr olds; (b) connection density change in the three age groups; (c) the histogram of the normalized anatomical distance associated with the significant connections in each age group's network; (d) comparison of the anatomical distances associated with connections showing age-dependent increase/ decrease in strength. Increasing connections with age (red) consistently possess longer anatomical distances than decreasing connections (blue, $p<1 \mathrm{e}-10$ from 0 to 1 and $p=1 \mathrm{e}-6$ from 1 to 2 ).

cyan-colored module spans across the frontal and temporal lobes; while the blue-colored module is distributed between the frontal and parietal lobes in $2 \mathrm{yr}$ olds, demonstrating a largely distributed functional modular structure. The apparent increase of long-range connections (shown in Fig. 10) highlights the modular structure changes from local to distributed network during the first two years of life.

One of the main characteristics of graph theory analysis is the determination of brain functional hubs for information transfer. The brain's functional hubs (top 10 regions showing highest betweenness centrality values at cost $15 \%$ ) demonstrate an interesting evolution pattern during the first two years of life (see Fig. 11). There are two apparent clusters of hubs, anterior and posterior clusters, in neonates, suggesting that major functional developments may have 


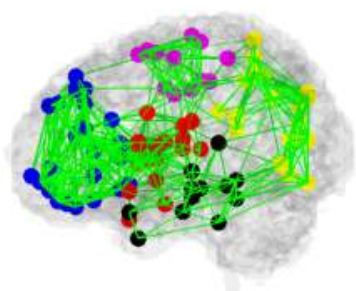

Neonates

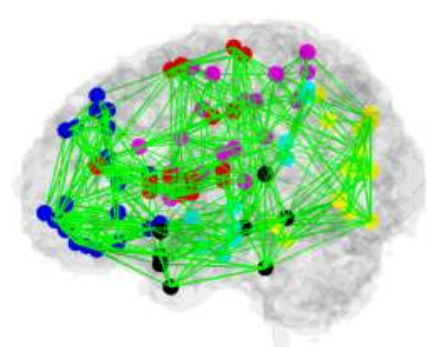

1-year-old

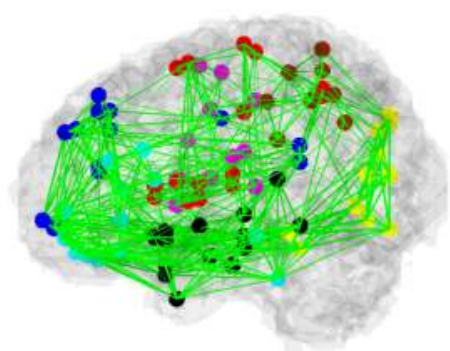

2-year-old

Figure 10. Changes of modular structure during the first two years of life. Different colors represent different modules in each age group.

already occurred in both the frontal and occipital lobes. In addition, most of the connections between the hubs are interhemispherical with no clear connections between the two clusters. In $1 \mathrm{yr}$ olds, the hubs are well connected and more anatomically centered with few hubs located in the anterior and posterior areas of the brain. Finally, the distribution of hubs becomes more spatially uniform in $2 \mathrm{yr}$ olds and the connections associated with the hubs provide a fairly uniform covering of the whole brain (Fig. 11).
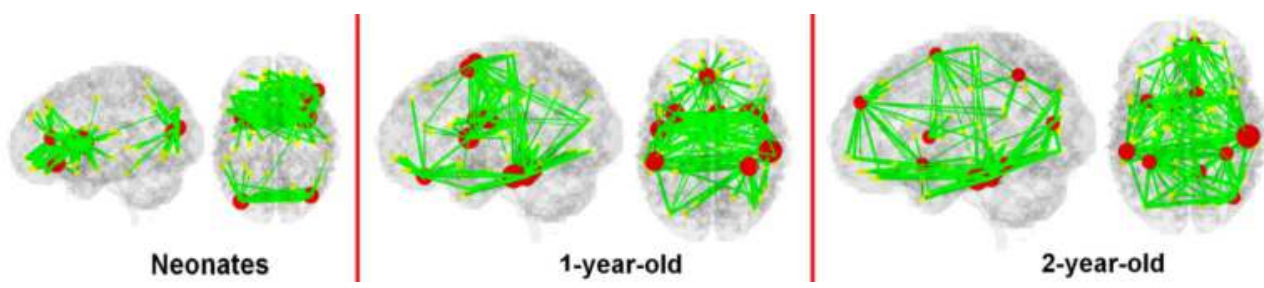

Figure 11. The distribution of functional hubs in the three age groups based on connection matrices at cost $15 \%$. Hub regions together with connections (at cost 15\%) are visualized on the corresponding brain surface.

Together, with the aforementioned brain functional network development during the first two years of life, it is highly plausible that brain efficacy for information transfer also undergoes a similar evolution process. To quantitatively examine the efficiency improvement resulting from such dramatic topological changes, local (LE) and global efficiency (GE) for the whole network were calculated for each individual subject at the same cost of $15 \%$. The results are presented in Fig. 12. Significant increases of LE and GE are observed from 3wks to 1yr of age (red asterisks, $\mathrm{p}=4 \mathrm{e}-7$ and $6 \mathrm{e}-8$ for LE and GE, respectively) but neither measure is statistically different between 1 and 2 yrs of age. Note when comparing neonates with $2 \mathrm{yr}$ olds, $2 \mathrm{yr}$ olds also demonstrate significantly higher LE ( $p=1 e-8)$ and GE ( $p=7 e-9)$.

In summary, the results reported in the literature have consistently indicated that the neonatal brain already exhibits a small-world network with reasonable information transfer efficiency 

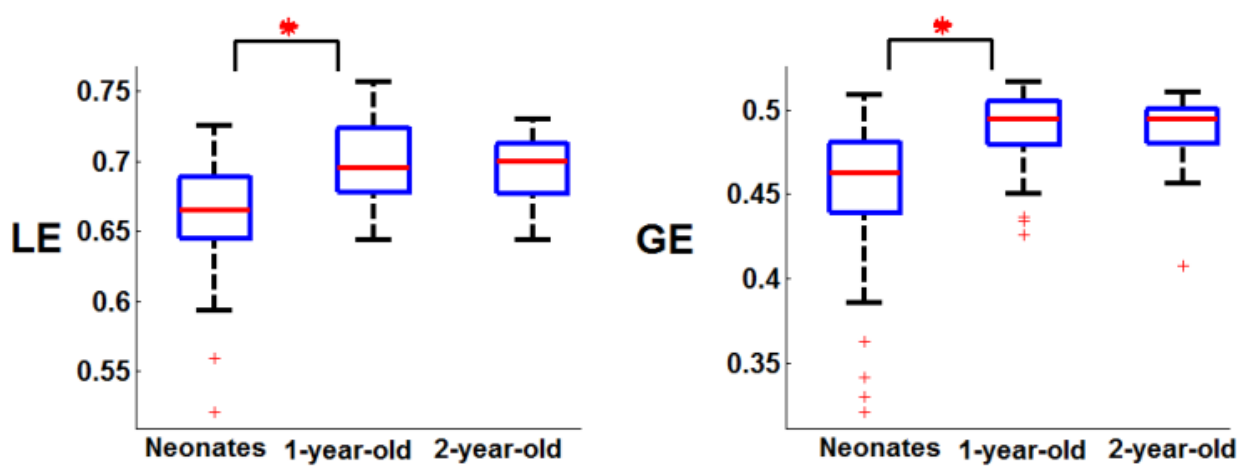

Figure 12. Quantitative measures of whole brain network's local efficiency (LE) and global efficiency (GE) at cost $15 \%$ based on individual subject's correlation matrices. Red asterisks represent significant difference at $\mathrm{p}<0.05$ (FDR corrected).

(Fransson et al., 2010; Gao et al., 2011). The well documented prenatal axonal development (Haynes et al., 2005) might set the foundation for an efficient connectivity network hence explains this finding. However, significant improvements of both LE and GE are observed from neonates to $1 \mathrm{yr}$ olds, whereas no statistical difference between 1 and $2 \mathrm{yr}$ olds is observed (Fig. 12). This non-linear developing pattern of brain wiring efficiency, particularly GE, is intriguing. Neonates demonstrate an anatomically ordered architecture (Fig. 10), in which neighboring clusters are largely sequentially connected, leading to a longer path length, while 1 and $2 y r$ olds show considerably better wired brain topologies with abundant bridging connections even between distant clusters, suggesting that anatomical long-range connections may play a critical role in the observed improvement. Indeed, quantitative compassions of connectivity strength show remarkable growth of longdistance connections (Fig. 9) strongly supporting the anatomical basis of network efficiency and suggesting that the establishment of a higher GE rests critically on the development of long-distance connections. An increase in long-distance connections has been reported when comparing school-aged children with adults (Fair et al., 2008; Fair et al., 2009; Kelly et al., 2009). Nevertheless, given the age differences between our study cohort and that reported in the literature, the biological underpinnings associated with the observed increased longrange connections most likely differ. Specifically, since the axonal properties (e.g., length, diameter, density) are most likely well established in school-aged children, the observed increase in long-range connectivity strength might primarily arise from the continuing myelination process, improving information transfer efficiency between distant brain regions (Tau and Peterson, 2010). In contrast, although some of the major long-range axonal connections (especially callosal fibers) are present in neonates (Fransson et al., 2007; Tau and Peterson, 2010), continuing axonal elongation/thickening is expected at this age to facilitate forming connections with other distant brain functional areas (e.g., in the anterior-posterior direction), especially during the first year of life (Tau and Peterson, 2010). Moreover, the myelination process is also documented to experience the most dramatic growth during the first two years of life (Gao et al., 2008; Haynes et al., 
2005; Tau and Peterson, 2010). Therefore, a combination of axonal growth and myelination likely accounts for the changes observed in our study.

The observation of no significant changes between 1yr and 2yr olds in whole brain smallworld properties echoes the findings by Supekar et al (Supekar et al., 2009) and Fair et al (Fair et al., 2009), who also failed to detect significant whole brain efficiency changes when comparing school-aged children with adults. However, significant regional wiring changes (LE/GE) have been documented in previous studies (Fair et al., 2009; Supekar et al., 2009; Gao et al., 2011). In considering these findings, it is plausible that the whole brain reaches an adultlike "smallworld" topology from 1-year-old on while after that although significant local changes continue to reshape the brain for the development of specific functions, the whole brain efficiency property remains un-disturbed.

\section{Explorations into developmental disorders}

Studies utilizing fcMRI to discern potential neuropathological underpinnings of various developmental disorders are emerging (Church et al., 2009; Di Martino et al., 2010; Dickstein et al., 2010; Fair et al., 2010; Liston et al., 2011; Qiu et al., 2010; Uddin et al., 2008). For example, Church et al (Church et al., 2009) examined youth subjects (aged between 9 to 15) with Tourette syndrome (TS), a developmental disorder characterized by irresistible stereotyped movements and vocalizations called "tics". They specifically evaluated functional connectivity associated with two putative control networks - a "fronto-parietal network" likely involved in online control and a "cingulo-opercular network" important for set-maintenance. Specifically, they found that most of the connections with abnormal connection strengths (less-matured) were associated with the fronto-parietal network indicating more pronounced disruption of adaptive online control function in TS subjects. Di Martino et al (Di Martino et al., 2010) investigated autism spectrum disorders (ASD), a developmental disorder characterized by impaired social interaction and communication. They compared the striatal functional connectivity between children with ASD (aged between 7-and 13-year-old) and normal controls and found widespread hyper-connectivity among all striatal regions as well as between striatal regions and other associative regions such as insular and right superior temporal gyrus. Based on these findings, they postulated that developmental derangement rather than immaturity of functional circuits might underlie ASD. Dickstein et al (Dickstein et al., 2010) studied pediatric bipolar disorder (BD) in youth subjects between the age of seven and seventeen and found greater negative functional connectivity between left dorsal lateral prefrontal cortex and right superior temporal gyrus in BD subjects when compared with normal controls, indicating abnormal development of this important circuit for working memory and learning.

Attention-deficit/hyperactivity disorder (ADHD) characterized by attentional problems and hyperactivity has also attracted great interest in resting-state fMRI studies (Liston et al., 2011). Among these, Fair et al (Fair et al., 2010) examined the functional connectivity within the default network and found generally reduced connectivity in children with ADHD comparing 
with normal controls, reflecting atypical circuit maturation of this network. Interestingly, using the independent component analysis approach, Qiu et al (Qiu et al., 2010) reported both decreased and increased functional connectivity within this network (i.e., increased functional connectivity in anterior cingulate cortex, posterior cingulate cortex, lateral prefrontal cortex, precuneus and thalamus but increased connectivity in posterior medial frontal cortex). In a separate study, Uddin et al (Uddin et al., 2008) also found decreased network homogeneity in the precuneus node of the default network. Besides default network, other studies targeting at $\mathrm{ADHD}$ have also investigated into abnormal functional connectivity in other parts of the brain. For a recent review of fcMRI studies on ADHD, please refer to Liston et al (Liston et al., 2011).

As described above, most of existing studies focused on the population of children no younger than 7-year-old. However, the first two years of life is perhaps a critical time period where certain developmental disorders start to develop such as autism syndrome disorder ASD (Levy et al., 2009). Other disorders such as ADHD may have a late onset as school-aged children but the underlying neurophysiologic changes may occur well before the onset of behavioral symptoms (Ilott et al., 2010; Kieling et al., 2008). Hence the understanding of the underlying neuropathology during the first years of life is vital to early diagnosis and potential treatment of such disorders. As a result, resting-state fMRI studies focusing on a much younger age period are highly warranted for successful delineation of the underlying neurophysiological mechanisms of different developmental disorders. In addition, most of the existing studies focus on specific circuits/connections, potentially limiting the findings of novel abnormal circuits/connections that may underlie such disorders. Therefore, more studies with an exploratory nature are greatly needed in future research.

\section{Technical issues in the application of fcMRI for early brain development}

There are potential issues that are of particular importance for the utilization of fcMRI in the developmental brain. The first and foremost is the definition of ROIs for pediatric subjects, especially for those younger than 1 year of age when both brain's structural morphology and functional organization is largely different from its matured configuration. The current practice of applying either a parcellation template or functional activation coordinates from adult studies to the pediatric populations facilitates direct comparison between the two populations. However, such a practice may lead to potential bias against pediatric subjects given the known organizational difference. To a certain extent, this may be a circular problem since it is this development related reorganization that we are studying. Fortunately, the utilization of a data-driven method such as ICA could potentially alleviate this problem given that no prior ROIs are needed. However, as mentioned previously, the choice of components reflecting functional connectivity is subjective. Thus, quantitative comparisons of connection strength are less straightforward for ICA-based approaches. Clearly, methodological improvements are greatly needed to solve such issues. 
But before that, developmental studies should choose different strategies according to the specific question(s) involved and the results should be interpreted with these issues in mind.

Viewing the brain's functional organization at the network level has been shown to be important in brain disease studies (Greicius et al., 2004; Seeley et al., 2009). For example, Seely et al (Seeley et al., 2009) proposed the "network degeneration hypothesis" where they reported that neurodegeneration may be related to network-level dysfunction and suggested the need for developing new network-based diagnostic assays. Logically, the interaction between different functional networks might also have significant influence on the developmental process. Different methods aimed at directly quantifying network-level interactions are emerging. Jafri et al (Jafri et al., 2008) described a method to calculate the functional connectivity between networks defined by spatial ICA and examined the differences between Schizophrenia and normal subjects. Gao et al (Gao et al., 2010) have proposed a different method based on canonical correlation to directly quantify the mutual dependence between two functional networks and applied it to delineate the dynamics of network level interactions under different brain states. In the future, we expect an increasing interest in both the development and application of network based analysis due to the intrinsic organization of the brain as individual functional modules.

Finally, in most cases the infants are sleeping inside the scanner with or without sedation in developmental investigations. The effect of sedation on resulting connectivity measurement should be better addressed. Even without sedation, different stages of sleep could also have an effect on the resulting functional connectivity measures. Therefore, studies incorporating sleep stage monitoring (e.g., through simultaneous EEG recording) would be beneficial to minimize experimental variability.

\section{Conclusion}

In this chapter, we have reviewed several results delineating the normal brain functional development process during the first two years of life using resting state functional connectivity. The application of fcMRI in various developmental disorders was also discussed. The uncertainty of the physiological underpinnings of fcMRI signal, as well as other technical issues related to its practical application are also briefly discussed pointing to the pressing need for improvement in future studies. The results support the notion that the brain's primary functional networks are in place and partially functioning immediately after birth, but the maturation of higher order cognitive networks takes prolonged experience based tuning during postnatal life. Moreover, dramatic evolution of the brain's functional topology is observed during this critical period, which greatly changes the brain's modular structure and distribution of hubs, as well as improves whole brain information transfer efficiency at both local and global levels. Finally, the evidence shows that less maturation and/or derangement of certain functional circuits might underlie different developmental disorders. 


\section{Author details}

Wei Gao ${ }^{1}$, Hongtu Zhu², Kelly S. Giovanello ${ }^{3}$, J. Keith Smith", Dinggang Shen ${ }^{1}$, John H. Gilmore ${ }^{5}$ and Weili Lin ${ }^{1}$

1 Department of Radiology and Biomedical Research Imaging Center, UNC-Chapel Hill, USA

2 Department of Biostatistics and Biomedical Research Imaging Center, UNC-Chapel Hill, USA

3 Department of Psychology and Biomedical Research Imaging Center, UNC-Chapel Hill, USA

4 Department of Radiology, UNC-Chapel Hill, USA

5 Department of Psychiatry, UNC-Chapel Hill, USA

\section{References}

[1] Achard, S., Bullmore, E., 2007. Efficiency and cost of economical brain functional networks. PLoS Comput Biol 3, e17.

[2] Achard, S., Salvador, R., Whitcher, B., Suckling, J., Bullmore, E., 2006. A resilient, low-frequency, small-world human brain functional network with highly connected association cortical hubs. J Neurosci 26, 63-72.

[3] Akhtar, N., \& Tomasello, M., 2000. The social nature of words and word learning. Oxford University Press., Oxford.

[4] Amsterdam, 1972. Mirror self-image reactions before age two. Developmental Psychology. Psychology 5, 297-305.

[5] Anderson, A.W., Marois, R., Colson, E.R., Peterson, B.S., Duncan, C.C., Ehrenkranz, R.A., Schneider, K.C., Gore, J.C., Ment, L.R., 2001. Neonatal auditory activation detected by functional magnetic resonance imaging. Magn Reson Imaging 19, 1-5.

[6] Andrews-Hanna, J.R., Reidler, J.S., Sepulcre, J., Poulin, R., Buckner, R.L., 2010. Functional-Anatomic Fractionation of the Brain's Default Network. Neuron 65, 550-562.

[7] Baldwin, D.A., 1993. Early referential understanding: young children' s ability to recognize referential acts for what they are. Developmental Psychology 29, 832-843.

[8] Bandettini, P.A., Wong, E.C., Hinks, R.S., Tikofsky, R.S., Hyde, J.S., 1992. Time course EPI of human brain function during task activation. Magn Reson Med 25, 390-397. 
[9] Barabasi, A.L., Albert, R., 1999. Emergence of scaling in random networks. Science 286, 509-512.

[10] Belliveau, J.W., Kennedy, D.N., Jr., McKinstry, R.C., Buchbinder, B.R., Weisskoff, R.M., Cohen, M.S., Vevea, J.M., Brady, T.J., Rosen, B.R., 1991. Functional mapping of the human visual cortex by magnetic resonance imaging. Science 254, 716-719.

[11] Biswal, B., Yetkin, F.Z., Haughton, V.M., Hyde, J.S., 1995. Functional connectivity in the motor cortex of resting human brain using echo-planar MRI. Magn Reson Med 34, 537-541.

[12] Biswal, B.B., Mennes, M., Zuo, X.N., Gohel, S., Kelly, C., Smith, S.M., Beckmann, C.F., Adelstein, J.S., Buckner, R.L., Colcombe, S., Dogonowski, A.M., Ernst, M., Fair, D., Hampson, M., Hoptman, M.J., Hyde, J.S., Kiviniemi, V.J., Kotter, R., Li, S.J., Lin, C.P., Lowe, M.J., Mackay, C., Madden, D.J., Madsen, K.H., Margulies, D.S., Mayberg, H.S., McMahon, K., Monk, C.S., Mostofsky, S.H., Nagel, B.J., Pekar, J.J., Peltier, S.J., Petersen, S.E., Riedl, V., Rombouts, S.A., Rypma, B., Schlaggar, B.L., Schmidt, S., Seidler, R.D., Siegle, G.J., Sorg, C., Teng, G.J., Veijola, J., Villringer, A., Walter, M., Wang, L., Weng, X.C., Whitfield-Gabrieli, S., Williamson, P., Windischberger, C., Zang, Y.F., Zhang, H.Y., Castellanos, F.X., Milham, M.P., 2010. Toward discovery science of human brain function. Proc Natl Acad Sci U S A 107, 4734-4739.

[13] Born, P., Leth, H., Miranda, M.J., Rostrup, E., Stensgaard, A., Peitersen, B., Larsson, H.B., Lou, H.C., 1998. Visual activation in infants and young children studied by functional magnetic resonance imaging. Pediatr Res 44, 578-583.

[14] Born, P., Rostrup, E., Leth, H., Peitersen, B., Lou, H.C., 1996. Change of visually induced cortical activation patterns during development. Lancet 347, 543.

[15] Brandes, U., 2001. A faster algorithm for betweenness centrality. J Math Sociol 25, 163-177.

[16] Buckner, R.L., Andrews-Hanna, J.R., Schacter, D.L., 2008. The brain's default network: anatomy, function, and relevance to disease. Ann N Y Acad Sci 1124, 1-38.

[17] Buckner, R.L., Carroll, D.C., 2007. Self-projection and the brain. Trends Cogn Sci 11, 49-57.

[18] Bullmore, E., Sporns, O., 2009. Complex brain networks: graph theoretical analysis of structural and functional systems. Nat Rev Neurosci 10, 186-198.

[19] Cepeda, N.J., Kramer, A.F., Gonzalez de Sather, J.C., 2001. Changes in executive control across the life span: examination of task-switching performance. Dev Psychol 37, 715-730.

[20] Chang, C., Glover, G.H., Time-frequency dynamics of resting-state brain connectivity measured with fMRI. Neuroimage 50, 81-98. 
[21] Church, J.A., Fair, D.A., Dosenbach, N.U., Cohen, A.L., Miezin, F.M., Petersen, S.E., Schlaggar, B.L., 2009. Control networks in paediatric Tourette syndrome show immature and anomalous patterns of functional connectivity. Brain 132, 225-238.

[22] Collins, D.1., Holmes, C.J., Peters, T.M., Evans, A.C., 1995. Automatic 3-D modelbased neuroanatomical segmentation. Hum Brain Mapp 3, 190-208.

[23] Conel, J.L., 1939. The Postnatal Development of Human Cerebral Cortex. Harvard University Press, Cambridge.

[24] Damoiseaux, J.S., Rombouts, S.A., Barkhof, F., Scheltens, P., Stam, C.J., Smith, S.M., Beckmann, C.F., 2006. Consistent resting-state networks across healthy subjects. Proc Natl Acad Sci U S A 103, 13848-13853.

[25] De Martino, F., Gentile, F., Esposito, F., Balsi, M., Di Salle, F., Goebel, R., Formisano, E., 2007. Classification of fMRI independent components using IC-fingerprints and support vector machine classifiers. Neuroimage 34, 177-194.

[26] DeCasper, A.J., Fifer, W.P., 1980. Of human bonding: newborns prefer their mothers' voices. Science 208, 1174-1176.

[27] Dehaene-Lambertz, G., Dehaene, S., Hertz-Pannier, L., 2002. Functional neuroimaging of speech perception in infants. Science 298, 2013-2015.

[28] Di Martino, A., Kelly, C., Grzadzinski, R., Zuo, X.N., Mennes, M., Mairena, M.A., Lord, C., Castellanos, F.X., Milham, M.P., 2010. Aberrant striatal functional connectivity in children with autism. Biol Psychiatry 69, 847-856.

[29] Dickstein, D.P., Gorrostieta, C., Ombao, H., Goldberg, L.D., Brazel, A.C., Gable, C.J., Kelly, C., Gee, D.G., Zuo, X.N., Castellanos, F.X., Milham, M.P., 2010. Fronto-temporal spontaneous resting state functional connectivity in pediatric bipolar disorder. Biol Psychiatry 68, 839-846.

[30] Diesendruck, G., Markson, L., Akhtar, N., Reudor, A., 2004. Two-year-olds' sensitivity to speakers' intent: an alternative account of Samuelson and Smith. Dev Sci 7, 33-41.

[31] Ebbels, T.M., Buxton, B.F., Jones, D.T., 2006. springScape: visualisation of microarray and contextual bioinformatic data using spring embedding and an 'information landscape'. Bioinformatics 22, e99-107.

[32] Fair, D.A., Cohen, A.L., Dosenbach, N.U., Church, J.A., Miezin, F.M., Barch, D.M., Raichle, M.E., Petersen, S.E., Schlaggar, B.L., 2008. The maturing architecture of the brain's default network. Proc Natl Acad Sci U S A 105, 4028-4032.

[33] Fair, D.A., Cohen, A.L., Power, J.D., Dosenbach, N.U., Church, J.A., Miezin, F.M., Schlaggar, B.L., Petersen, S.E., 2009. Functional brain networks develop from a "local to distributed" organization. PLoS Comput Biol 5, e1000381. 
[34] Fair, D.A., Posner, J., Nagel, B.J., Bathula, D., Dias, T.G., Mills, K.L., Blythe, M.S., Giwa, A., Schmitt, C.F., Nigg, J.T., 2010. Atypical default network connectivity in youth with attention-deficit/hyperactivity disorder. Biol Psychiatry 68, 1084-1091.

[35] Feinberg, D.A., Moeller, S., Smith, S.M., Auerbach, E., Ramanna, S., Glasser, M.F., Miller, K.L., Ugurbil, K., Yacoub, E., 2010. Multiplexed echo planar imaging for subsecond whole brain FMRI and fast diffusion imaging. PLoS One 5, e15710.

[36] Fivush, R., Hamond, N.R, 1990. Autobiographical memory across the preschool years: Toward reconcepualizing childhood amnesia. In: J.A.Hudson, R.F.a. (Ed.), Knowing and remembering in young children. Cambridge Univ. Press, New York:

[37] Fornito, A., Zalesky, A., Bullmore, E.T., 2010. Network scaling effects in graph analytic studies of human resting-state FMRI data. Front Syst Neurosci 4, 22.

[38] Fox, M.D., Corbetta, M., Snyder, A.Z., Vincent, J.L., Raichle, M.E., 2006. Spontaneous neuronal activity distinguishes human dorsal and ventral attention systems. Proc Natl Acad Sci U S A 103, 10046-10051.

[39] Fox, M.D., Zhang, D., Snyder, A.Z., Raichle, M.E., 2009. The global signal and observed anticorrelated resting state brain networks. J Neurophysiol 101, 3270-3283.

[40] Fransson, P., Aden, U., Blennow, M., Lagercrantz, H., 2010. The Functional Architecture of the Infant Brain as Revealed by Resting-State fMRI. Cereb Cortex.

[41] Fransson, P., Skiold, B., Horsch, S., Nordell, A., Blennow, M., Lagercrantz, H., Aden, U., 2007. Resting-state networks in the infant brain. Proc Natl Acad Sci U S A 104, 15531-15536.

[42] Fulford, J., Vadeyar, S.H., Dodampahala, S.H., Moore, R.J., Young, P., Baker, P.N., James, D.K., Gowland, P.A., 2003. Fetal brain activity in response to a visual stimulus. Hum Brain Mapp 20, 239-245.

[43] Gao, W., Lin, W., 2011. Frontal parietal control network regulates the anti-correlated default and dorsal attention networks. Hum Brain Mapp (In Press).

[44] Gao, W., Lin, W., Chen, Y., Gerig, G., Smith, J.K., Jewells, V., Gilmore, J.H., 2008. Temporal and Spatial Development of Axonal Maturation and Myelination of White Matter in the Developing Brain. AJNR Am J Neuroradiol.

[45] Gao, W., Zhu, H., Giovanello, K., Lin, W., 2010. Multivariate network-level approach to detect interactions between large-scale functional systems. Med Image Comput Comput Assist Interv 13, 298-305.

[46] Gao, W., Zhu, H., Giovanello, K.S., Smith, J.K., Shen, D., Gilmore, J.H., Lin, W., 2009. Evidence on the emergence of the brain's default network from 2-week-old to 2-yearold healthy pediatric subjects. Proc Natl Acad Sci U S A 106, 6790-6795. 
[47] Greicius, M.D., Krasnow, B., Reiss, A.L., Menon, V., 2003. Functional connectivity in the resting brain: a network analysis of the default mode hypothesis. Proc Natl Acad Sci U S A 100, 253-258.

[48] Greicius, M.D., Menon, V., 2004. Default-mode activity during a passive sensory task: uncoupled from deactivation but impacting activation. J Cogn Neurosci 16, 1484-1492.

[49] Greicius, M.D., Srivastava, G., Reiss, A.L., Menon, V., 2004. Default-mode network activity distinguishes Alzheimer's disease from healthy aging: evidence from functional MRI. Proc Natl Acad Sci U S A 101, 4637-4642.

[50] Gusnard, D.A., Akbudak, E., Shulman, G.L., Raichle, M.E., 2001. Medial prefrontal cortex and self-referential mental activity: relation to a default mode of brain function. Proc Natl Acad Sci U S A 98, 4259-4264.

[51] Gusnard, D.A., Raichle, M.E., 2001. Searching for a baseline: functional imaging and the resting human brain. Nat Rev Neurosci 2, 685-694.

[52] Haynes, R.L., Borenstein, N.S., Desilva, T.M., Folkerth, R.D., Liu, L.G., Volpe, J.J., Kinney, H.C., 2005. Axonal development in the cerebral white matter of the human fetus and infant. J Comp Neurol 484, 156-167.

[53] He, B.J., Snyder, A.Z., Zempel, J.M., Smyth, M.D., Raichle, M.E., 2008. Electrophysiological correlates of the brain's intrinsic large-scale functional architecture. Proc Natl Acad Sci U S A 105, 16039-16044.

[54] Hilgetag, C.C., Burns, G.A., O'Neill, M.A., Scannell, J.W., Young, M.P., 2000. Anatomical connectivity defines the organization of clusters of cortical areas in the macaque monkey and the cat. Philos Trans R Soc Lond B Biol Sci 355, 91-110.

[55] Hykin, J., Moore, R., Duncan, K., Clare, S., Baker, P., Johnson, I., Bowtell, R., Mansfield, P., Gowland, P., 1999. Fetal brain activity demonstrated by functional magnetic resonance imaging. Lancet 354, 645-646.

[56] Ilott, N.E., Saudino, K.J., Asherson, P., 2010. Genetic influences on attention deficit hyperactivity disorder symptoms from age 2 to 3 : a quantitative and molecular genetic investigation. BMC Psychiatry 10, 102.

[57] Jafri, M.J., Pearlson, G.D., Stevens, M., Calhoun, V.D., 2008. A method for functional network connectivity among spatially independent resting-state components in schizophrenia. Neuroimage 39, 1666-1681.

[58] Joel, S.E., Caffo, B.S., van Zijl, P.C., Pekar, J.J., 2011. On the relationship between seed-based and ICA-based measures of functional connectivity. Magn Reson Med.

[59] Johnson, M.H., 2000. Functional brain development in infants: elements of an interactive specialization framework. Child Dev 71, 75-81. 
[60] Johnson, M.H., 2001. Functional brain development in humans. Nat Rev Neurosci 2, 475-483.

[61] Joseph, R., 1999. Fetal Brain \& Cognitive Development. Developmental Review 20, 81-98.

[62] Kelly, A.M., Di Martino, A., Uddin, L.Q., Shehzad, Z., Gee, D.G., Reiss, P.T., Margulies, D.S., Castellanos, F.X., Milham, M.P., 2009. Development of anterior cingulate functional connectivity from late childhood to early adulthood. Cereb Cortex 19, 640-657.

[63] Kieling, C., Goncalves, R.R., Tannock, R., Castellanos, F.X., 2008. Neurobiology of attention deficit hyperactivity disorder. Child Adolesc Psychiatr Clin N Am 17, 285-307, viii.

[64] Kolata, G., 1984. Studying learning in the womb. Science 225, 302-303.

[65] Latora, V., Marchiori, M., 2001. Efficient behavior of small-world networks. Phys Rev Lett 87, 198701.

[66] Levy, S.E., Mandell, D.S., Schultz, R.T., 2009. Autism. Lancet 374, 1627-1638.

[67] Lin, W., Zhu, Q., Gao, W., Chen, Y., Toh, C.H., Styner, M., Gerig, G., Smith, J.K., Biswal, B., Gilmore, J.H., 2008. Functional connectivity MR imaging reveals cortical functional connectivity in the developing brain. AJNR Am J Neuroradiol 29, 1883-1889.

[68] Liston, C., Cohen, M.M., Teslovich, T., Levenson, D., Casey, B.J., 2011. Atypical prefrontal connectivity in attention-deficit/hyperactivity disorder: pathway to disease or pathological end point? Biol Psychiatry 69, 1168-1177.

[69] Liu, Y., Liang, M., Zhou, Y., He, Y., Hao, Y., Song, M., Yu, C., Liu, H., Liu, Z., Jiang, T., 2008. Disrupted small-world networks in schizophrenia. Brain 131, 945-961.

[70] Logothetis, N.K., 2003. The underpinnings of the BOLD functional magnetic resonance imaging signal. J Neurosci 23, 3963-3971.

[71] Logothetis, N.K., Pauls, J., Augath, M., Trinath, T., Oeltermann, A., 2001. Neurophysiological investigation of the basis of the fMRI signal. Nature 412, 150-157.

[72] Lowe, M.J., Mock, B.J., Sorenson, J.A., 1998. Functional connectivity in single and multislice echoplanar imaging using resting-state fluctuations. Neuroimage 7, 119-132.

[73] Murphy, K., Birn, R.M., Handwerker, D.A., Jones, T.B., Bandettini, P.A., 2009. The impact of global signal regression on resting state correlations: are anti-correlated networks introduced? Neuroimage 44, 893-905. 
[74] Neumann, J., von Cramon, D.Y., Forstmann, B.U., Zysset, S., Lohmann, G., 2006. The parcellation of cortical areas using replicator dynamics in fMRI. Neuroimage 32, 208-219.

[75] Newman, M.E., 2006. Modularity and community structure in networks. Proc Natl Acad Sci U S A 103, 8577-8582.

[76] Nir, Y., Mukamel, R., Dinstein, I., Privman, E., Harel, M., Fisch, L., Gelbard-Sagiv, H., Kipervasser, S., Andelman, F., Neufeld, M.Y., Kramer, U., Arieli, A., Fried, I., Malach, R., 2008. Interhemispheric correlations of slow spontaneous neuronal fluctuations revealed in human sensory cortex. Nat Neurosci 11, 1100-1108.

[77] Ogawa, S., Lee, T.M., Nayak, A.S., Glynn, P., 1990. Oxygenation-sensitive contrast in magnetic resonance image of rodent brain at high magnetic fields. Magn Reson Med 14, 68-78.

[78] Perlbarg, V., Bellec, P., Anton, J.L., Pelegrini-Issac, M., Doyon, J., Benali, H., 2007. CORSICA: correction of structured noise in fMRI by automatic identification of ICA components. Magn Reson Imaging 25, 35-46.

[79] Qiu, M.G., Ye, Z., Li, Q.Y., Liu, G.J., Xie, B., Wang, J., 2010. Changes of Brain Structure and Function in ADHD Children. Brain Topogr.

[80] Raichle, M.E., MacLeod, A.M., Snyder, A.Z., Powers, W.J., Gusnard, D.A., Shulman, G.L., 2001. A default mode of brain function. Proc Natl Acad Sci U S A 98, 676-682.

[81] Raichle, M.E., Mintun, M.A., 2006. Brain work and brain imaging. Annu Rev Neurosci $29,449-476$.

[82] Salvador, R., Suckling, J., Coleman, M., Pickard, J. D., Menon, D., Bullmore,, E., 2005. Neurophysiological architecture of functional magnetic resonance images of human brain. Cerebral Cortex 34, 387-413.

[83] Seeley, W.W., Crawford, R.K., Zhou, J., Miller, B.L., Greicius, M.D., 2009. Neurodegenerative diseases target large-scale human brain networks. Neuron 62, 42-52.

[84] Shmuel, A., Leopold, D.A., 2008. Neuronal correlates of spontaneous fluctuations in fMRI signals in monkey visual cortex: Implications for functional connectivity at rest. Hum Brain Mapp 29, 751-761.

[85] Shulman GL, F.J., Corbetta M, Buckner RL, Miezin FM, 1997. Common blood flow changes across visual tasks: II.: decreases in cerebral cortex. J. Cogn. Neurosci 9, 648-663.

[86] Smyser, C.D., Inder, T.E., Shimony, J.S., Hill, J.E., Degnan, A.J., Snyder, A.Z., Neil, J.J., 2010. Longitudinal Analysis of Neural Network Development in Preterm Infants. Cereb Cortex.

[87] Supekar, K., Musen, M., Menon, V., 2009. Development of large-scale functional brain networks in children. PLoS Biol 7, e1000157. 
[88] Tau, G.Z., Peterson, B.S., 2010. Normal development of brain circuits. Neuropsychopharmacology 35, 147-168.

[89] Tohka, J., Foerde, K., Aron, A.R., Tom, S.M., Toga, A.W., Poldrack, R.A., 2008. Automatic independent component labeling for artifact removal in fMRI. Neuroimage 39, $1227-1245$.

[90] Tomasello, M., Strosberg, R., Akhtar, N., 1996. Eighteen-month-old children learn words in non-ostensive contexts. J Child Lang 23, 157-176.

[91] Tzourio-Mazoyer, N., Landeau, B., Papathanassiou, D., Crivello, F., Etard, O., Delcroix, N., Mazoyer, B., Joliot, M., 2002. Automated anatomical labeling of activations in SPM using a macroscopic anatomical parcellation of the MNI MRI single-subject brain. Neuroimage 15, 273-289.

[92] Uddin, L.Q., Kelly, A.M., Biswal, B.B., Margulies, D.S., Shehzad, Z., Shaw, D., Ghaffari, M., Rotrosen, J., Adler, L.A., Castellanos, F.X., Milham, M.P., 2008. Network homogeneity reveals decreased integrity of default-mode network in ADHD. J Neurosci Methods 169, 249-254.

[93] Van Dijk, K.R., Hedden, T., Venkataraman, A., Evans, K.C., Lazar, S.W., Buckner, R.L., 2010. Intrinsic functional connectivity as a tool for human connectomics: theory, properties, and optimization. J Neurophysiol 103, 297-321.

[94] Vincent, J.L., Kahn, I., Snyder, A.Z., Raichle, M.E., Buckner, R.L., 2008. Evidence for a frontoparietal control system revealed by intrinsic functional connectivity. J Neurophysiol 100, 3328-3342.

[95] Vincent, J.L., Snyder, A.Z., Fox, M.D., Shannon, B.J., Andrews, J.R., Raichle, M.E., Buckner, R.L., 2006. Coherent spontaneous activity identifies a hippocampal-parietal memory network. J Neurophysiol 96, 3517-3531.

[96] Wang, J., Wang, L., Zang, Y., Yang, H., Tang, H., Gong, Q., Chen, Z., Zhu, C., He, Y., 2009. Parcellation-dependent small-world brain functional networks: a resting-state fMRI study. Hum Brain Mapp 30, 1511-1523.

[97] Watts, D.J., Strogatz, S.H., 1998. Collective dynamics of 'small-world' networks. Nature $393,440-442$.

[98] Whitlow, C.T., Casanova, R., Maldjian, J.A., 2011. Effect of resting-state functional MR imaging duration on stability of graph theory metrics of brain network connectivity. Radiology 259, 516-524. 
